\title{
Evaluación de la estabilidad de las labores y pilar corona en las minas abandonadas de S'Argentera (Ibiza, España) combinando clasificaciones geomecánicas, métodos empíricos y análisis numérico - enfocado a su posible aprovechamiento turístico
}

\author{
Luis Jordá-Bordehore ${ }^{(1)}$, Rafael Jordá-Bordehore ${ }^{(2)}$, Juan José Durán Valsero ${ }^{(3)}$ y \\ Paola Leonor Romero-Crespo(1)
}

(1) Escuela Superior Politécnica del Litoral, ESPOL, Facultad de Ingeniería en Ciencias de laTierra, Campus Gustavo Galindo Km 30.5 Vía Perimetral, P.O. Box 09-01-5863, Guayaquil, Ecuador ljorda@espol.edu.ec

(2) Rudnik Ciencias de laTierra S.L. Calle Cañada 5, 28720, Bustarviejo, Madrid, España

(3) Instituto Geológico y Minero de España, Rios Rosas 19, Madrid, España

\begin{abstract}
RESUMEN
Las minas de S'Argentera en Ibiza (Islas Baleares, España) son unas labores subterráneas de plomo argentífero abandonadas desde la primera década del siglo XX. Se ha analizado la estabilidad geotécnica de las cámaras y del pilar corona de las minas (sobre parte del cual pasa la carretera entre Santa Eulalia y San Carles de Peralta), dado que se está contemplando en un futuro permitir el acceso a la parte superior del conjunto de minas e iniciar un proyecto de rehabilitación turística de las labores subterráneas. Se ha evaluado la estabilidad combinando un enfoque empírico mediante el índice $Q$, método gráfico de estabilidad y ancho escalado, y métodos numéricos de elementos finitos para análisis tensodeformacional. El resultado del análisis muestra que las cámaras de las minas son estables pero algunas no poseen un adecuado factor de seguridad. Por lo tanto son necesarios algunos refuerzos y monitoreo puntuales. La modelización tensodeformacional en dos dimensiones arroja resultados realistas sobre la estabilidad actual de la cámara y los posibles desprendimientos ya producidos.
\end{abstract}

Palabras Clave: Clasificaciones geomecánicas, elementos finitos, geotecnia, mecánica de rocas, mina

\section{Stability assessment of the stopes and crown pillar of the S'Argentera abandoned mines (Ibiza, Spain) using geomechanical classifications, an empirical approach and numerical analysis focused on a possible tourist exploitation}

\author{
ABSTRACT
}

The argentiferous lead mines of S'Argentera in Ibiza (Balearic Islands, Spain) are some abandoned underground workings from the first decade of the $20^{\text {th }}$ century. The stability of the main stopes and the crown pillar of the mines has been evaluated - part of which is located below the road between Santa Eulalia and San Carles de Peralta. The possibility of allowing public access to the surface of the mining area and setting up a project of a "show mine" on the underground stopes and galleries is analysed. The stability is assessed with an empirical approach using the $O$ index, the scaled span method and a stability graph together with a numerical approach. Results show that the stopes of the mines are globally stable but some lack an adequate safety factor. Therefore some local reinforcements and monitoring are needed. The finite element 
Jordá-Bordehore, et al., 2017. Evaluación de la estabilidad de las labores y pilar... Boletín Geológico y Minero, 128 (1): 3-24

modelling in two dimensions yields realistic results on the current stope stability and possible rock falls which have already occurred.

Keywords: finite elements, geotechnics, geomechanical classifications, mine, rock mechanics

ABRIDGED ENGLISH VERSION

\section{Introduction and methods}

The mines of S'Argentera (from the Catalonian "La Platera") are a complex of underground argentiferous lead mines that have been abandoned since 1909. They are located in the proximities of the small picturesque village of San Carles on the island of Ibiza (Balearic Islands, Spain) in what is now a highly touristic area (Fig.1). There are documents testifying to the existence of the mines from the XIV century, but the height of their exploitation was between 1867 and 1909, when they were abandoned due to flooding in the richest part of the mine. Strangely, the complex is now completely dry and the ground water level is $20 \mathrm{~m}$ lower. Both the external and underground heritage form one of the most important mining relics of the Balearic Islands and the area is under study with the aim of turning it into a tourist attraction (Escandell Serra 2001). Topographic and geomechanical as well as cave exploration work has been underway since 2002 (Fig. 2).

The aim of this study is to apply an internationally accepted empirical method based on geomechanical classifications and numerical modelling to determine the stability of the crown pillars and shallow stopes, establishing a series of recommendations concerning the stability and possible actions to take for the particular case of the largest mine in the area, which passes under the road PM-810 between San Carles de Peralta and Santa Eulalia. In the event that access over an old mining area is proposed the degree of risk assumed must be established and whether the conditions of stability permit it to be considered as a freely accessible area or one to which the public is denied access.

The study carried out covered diverse investigations and analysis between 2010 and 2014:

- Geotechnical field work using geomechanical stations in the interior of the mine with the help of speleological techniques (descent via two old mine shafts, Fig. 2)

- Evaluation of the stability of the stopes by empirical methods $-Q$ index for $2 D$ analysis (Fig. 5) and stability graph method for 3D (Fig. 8 and Fig. 9).

- Stability assessment of the crown pillar using the 'scaled span' approach (Fig. 7).

- Seismic refraction profiles with the aim of determining ground thickness - altered rock and inferring the geomechanical parameters Q-Qc of the rock massif (Figs. 3 and 4).

- Numerical modelling and stress-displacement assessment - applying the Hoek and Brown criterion (Hoek et al., 2002) for the rock mass of the worst section of the larger stope - the west one (Figs. 11 and 12).

\section{Results and discussion}

Stability assessment using the $Q$ index $-Q$ graph approach (Table 1 and Fig. 5) suggests that, regarding the Excavation Support Ratio ESR - in the case that the stopes were accessible, some support or reinforcements in the stopes would be necessary and therefore recommended. The $Q$ index is also used in the other two empirical approaches of this work: the scaled span and stability graph methods. In this paper new sub-categories of the ESR are proposed (Table 2). They consider a possible touristic use of underground excavations such as mines and caves.

In most cases access to the abandoned mine workings for the purposes of setting up geomechanical stations is not feasible for safety reasons, and seismic refraction is the most reliable alternative for determining the soundness of the underlying rock mass. Seismic refraction was therefore used to check the quality values of the rock massif and to determine the rock ground contact, which delimits the real thickness of the crown pillar at $7 \mathrm{~m}$. (Fig.3). To correlate Vp (in $\mathrm{m} / \mathrm{s}$ ) with the rock quality Qc (the parameter Qc is $Q$ multiplied by the quotient $\sigma \mathrm{c} / 100)$ the graph in Fig. 4 (originally by Barton, 2002) was used. For seismic acquisition a 24- channel geometric seismograph and $5 \mathrm{~m}$ of geophone spacing with a total of 6 - 7 shot points were used.

Evaluation using the stability graph method suggests that both the east and west stopes are stable. The back of the east and the west stopes are closer to the instability area, therefore require more attention.

In the case of mining stopes with a clearly polyhedric form and which cannot be considered as an infinite gallery in 2D, an adapted geomechanical classification would be more appropriate and would better reflect the reality, such as the stability graph method (Figs. 8 and 9, table 6 and 7) instead of the classical $Q$ chart 
Jordá-Bordehore, et al., 2017. Evaluación de la estabilidad de las labores y pilar... Boletín Geológico y Minero, 128 (1): $3-24$

(Barton et al., 1974, ). As seen in Figure 10, all the faces of the stopes analyzed are found in the graph Stability Number $N^{\prime}$ vs Hydraulic Radius in the stable area, as might be expected of mining works that have been open for over 100 years and only present small occasional instabilities of a kinematic nature (wedges). As it is the largest face, the west stope back is the one that lies closest to the limit.

The crown pillar assessment using the scaled span method (Figs 6 and 7, Tables 3 and 4) indicate that certain reinforcements and ground surface monitoring is recommended if any access above the crown pillar is to be allowed.

The mine has been modelled in two dimensions, with a gravitational vertical tension type $\sigma v=\gamma \times h$, with $K O=1$ and boundary conditions with restrictions on the movement along the $Y$ axis in the vertical limits, free surface and restricted $X$ and $Y$ in the lower base (Fig. 13). Elements considered are triangular with three nodes. We have considered four stages including initial excavations and three mining excavations (Fig. 13). The results of yielded elements (Fig. 14) show that there is some yielding in the contact roof - the hanging wall the stope from the first excavation phase which progresses to the surface and some central parts of the span in the final stage in which the entire mining stope was excavated: this was the situation at the end of the exploitation of the mine in 1909. The total surface displacement lies under $4 \mathrm{~cm}$, which meant that in time of the excavation of the mine no surface risk was expected. There may have been slight ground motion when the stope was opened completely but nowadays it has stabilized. As for the maximum movement in the mine stope this is located in the in the middle of the span and reaches $19 \mathrm{~cm}$ (Fig.15) in a piece of the roof that was afterwards excavated, which faithfully reflects the observed reality: these blocks fell and currently no cracks are visible in the ceiling but blocks detached from the time of the exploitation are on the floor. As for the maximum stresses and stress concentration, tractions occur in the centre of the span that led to the fall of a block effect subsequently stabilized leading to a stable discharge arc (Fig. 16).

Evaluations by empirical methods, geomechanical classifications and a numerical approach indicate that the mine stopes and crown pillars are globally stable and are not expected to influence the surface. However some local instability has been detected, which means that some support will be required to allow visitors to get into the mine. It is recommended, given the touristic use the site is intended for, that a basic system of auscultation is constructed or installed and that there is a periodic survey of possible instabilities.

In the case of touristic usage of mining heritage, appropriate analysis of abandoned mines is essential, both of the stopes and their interaction with the surface. It must be determined whether access should be restricted (and/or they should be reinforced) or whether access should be prohibited. Modern studies using numerical methods tend to distance empirical methodologies, however these are extremely useful since the empirical charts and the conclusions they provide are backed up by numerous cases. Using just a few measurements both of the dimensions and of the logical geomechanical parameters, it is feasible to make a correct evaluation of stability, or at the least to know the graphic area we are in, stable or unstable (collapse). The validity of the conclusions of a numerical approach is often a compromise among economy, time for the analysis and the availability of reliable data. In many cases it is impossible to achieve this level of detail. The empirical methods are often a good compromise between economy, time of analysis and the availability of reliable data.

\section{Introducción}

Las minas de S'Argentera (del catalán "La Platera") son unas labores subterráneas de plomo argentífero de cierta entidad. Se localizan en las proximidades del pintoresco pueblo de San Carles en la isla de lbiza (Baleares, España) en una zona hoy día con mucho trasiego turístico (Fig. 1). Existen documentos que atestiguan su explotación desde el siglo XIV, pero la época de máximo apogeo fue entre 1867 hasta su abandono en 1909, debido a los problemas de inundación de la parte más rica del yacimiento - hoy en día curiosamente las labores están completamente secas y el nivel freático $20 \mathrm{~m}$ por debajo. El patrimonio tanto externo como subterráneo conforma uno de los vestigios mineros más importantes de las Islas Baleares y la zona se encuentra en fase de estudio para un aprovechamiento turístico (Escandell Serra 2001, Jordá et al 2011). Desde el año 2002 se llevan a cabo trabajos de topografía subterránea, geomecánica y exploración espeleológica (Fig. 2).

El objetivo del trabajo es poder responder, en este caso, a las siguientes preguntas:

- ¿Es estable el minado?

- ¿Es posible el acceso público a la zona?.

Para ello se va a aplicar una metodología empírica internacionalmente aceptada en base a clasificaciones geomecánicas para el estudio de estabilidad de pilares corona y de las cámaras someras, en particular de la labor minera de mayor envergadura de la zona - la cual pasa por debajo de la carretera PM-810 entre San Carles de Peralta y Santa Eulalia. En el caso de que sobre una antigua zona minada se permita 
Jordá-Bordehore, et al., 2017. Evaluación de la estabilidad de las labores y pilar... Boletín Geológico y Minero, 128 (1): 3-24

el acceso es preceptivo saber si las condiciones de estabilidad permiten considerarla como un área de acceso libre o restringido del público. Se ha modelizado la sección más desfavorable de la cámara de mayores dimensiones mediante elementos finitos en dos dimensiones.

Desde la década de los 70 del siglo $X X$ se han ido abandonando por agotamiento o altos costes operativos minas clásicas del Viejo Continente, lo cual unido a más de dos mil años de intensa minería, ha generado una enorme cantidad de pasivos mineros formados por labores superficiales. La proliferación de infraestructura y la ampliación de las zonas urbanizadas ha hecho que la minería abandonada suponga un importante riesgo para laciudadanía. No es una problemática exclusiva del viejo continente, aunque en Europa es muy llamativo el fenómeno. En otros continentes como Asia y América hay referencias de la preocupación de las autoridades por la problemática geotécnica que suponen las minas abandonadas (Carter y Miller 1995, Waltham et.al. 2014, Yong-Bok Jung et.al., 2014).

Para el análisis geotécnico de minas y obras subterráneas lo más común en las última dos décadas es combinar métodos empíricos y análisis tensodeformacional (Barton y Bieniaski 2008, Cornejo y Salvador 1997, Hoek et al 1995). Helm et.al., (2013) analizan la subsidencia urbana producida por minas abandonadas de carbón, en su caso el modelo empleado ha sido de diferencias finitas con código FLAC3D. Se han encontrado referencias a la modelización numérica de minas abandonadas de cara a un aprovechamiento turístico en las mina Monchi y Bustarviejo (Jordá et al 2010 y Jordá y Jordá, 2009 respectivamente). En el caso de la minería de carbón hay otras metodologías (Yong-Bok Jung, 2014) para analizar la susceptibilidad al hundimiento de los terrenos sobre minas. En el caso de minería somera en roca competente la metodología de ancho escalado de pilar corona está ampliamente aplicada en el mundo (Carter y Miller 1995, Carter 2014) y es una de las que se aplicó en la presente investigación. En el caso de la estabilidad de las cámaras en si mismas, el método gráfico de estabilidad (Nickson, 1992, Potvin 1988) es el más empelado a nivel mundial. Ambos se fundamentan en el índice $Q$ que junto con el Rock Mass Rating son las clasificaciones geomecánicas más extendidas en el mundo (Bieniawski 1989, Barton y Bieniawski, 2008)

El enfoque moderno y más completo es el de realizar una primera aproximación al problema mediante métodos empíricos que permitan encuadrar el problema y combinarlo con métodos numéricos para análisis más en profundidad y poder establecer los rangos y criterios para un posterior monitoreo del espacio subterráneo.

\section{Contexto geológico y descripción de las minas}

Se trata de un yacimiento paleo - kárstico en una mineralización de $\mathrm{Pb}-\mathrm{Zn}$ originada por procesos de alteración superficial en dolomías del Lías - Dogger (Arribas y Moro, 1985). La galena argentífera se encuentra diseminada entre capas de dolomías y margas arcillosas así como formando filoncillos y riñones; y como nódulos milimétricos en el propio relleno kárstico arcilloso. Dentro de las labores mineras de interior se aprecian tanto conductos naturales kársticos de techo como realces mineros efectuados en cavidades rellenas de arcillas rojas de descalcificación.

El entorno de las minas de S'Argentera está conformado principalmente por vestigios mineros subterráneos, que se desarrollan a profundidades variables entre unos escasos 2- $3 \mathrm{~m}$, hasta $20 \mathrm{~m}$ (Fig. 1). A lo largo de la zona minada y declarada Bien de Interés Cultural hay colapsos mineros someros de diversa índole (Fig.2) principalmente en labores muy someras y en roca de mala calidad, debidos en su mayoría a fallos en el pilar corona. En las minas de S'Argentera se encuentran principalmente 3 tipos de laboreo minero:

i. Zonas con roca de buena calidad y que se explotaron mediante cámaras abiertas de cierta entidad (hasta $6 \mathrm{~m}$ de vano y 19 a $59 \mathrm{~m}$ de longitud y 3 a 5 $\mathrm{m}$ de alto)

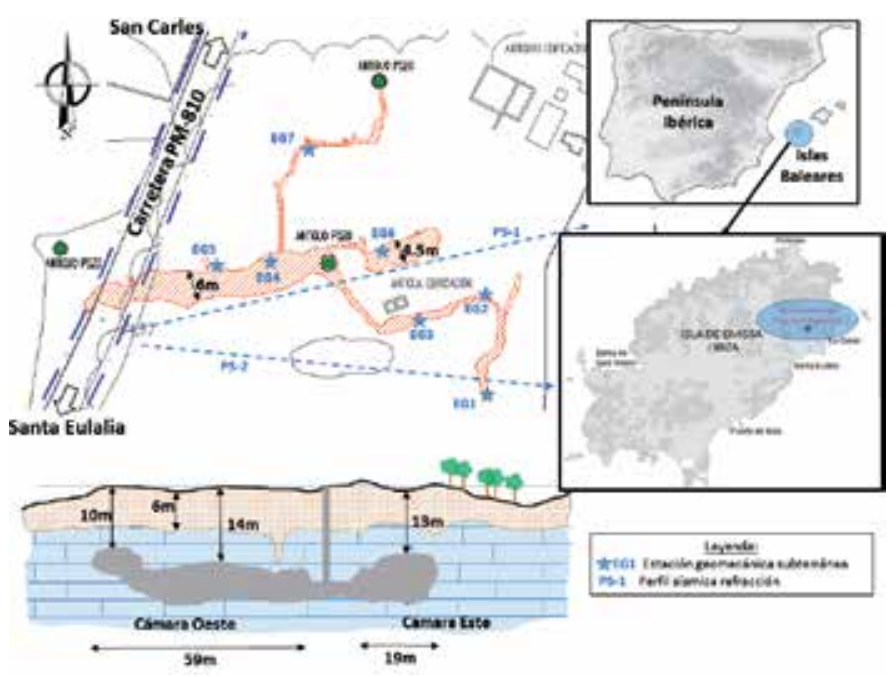

Figura 1. Localización de las labores mineras de S'Argentera a su paso bajo la carretera PM-810 (Ibiza, Islas Baleares).

Figure 1. Location of S'Argentera mining works crossing under the road PM-810 (Ibiza, Balearic islands). 
ii. Zonas de peor calidad donde se explotaba mediante una suerte de tajo largo, en las que se abrían los huecos de tamaño mínimo y se entibaba provisionalmente con madera de sabina y se rellenaban las labores explotadas con muros roca estéril. Estos minados también han perdurado en el tiempo (Fig.2c).

iii. Zonas muy someras con minados más antiguos (mediados S XIX) donde se ha apreciado una insuficiencia de sostenimiento y/o la degradación

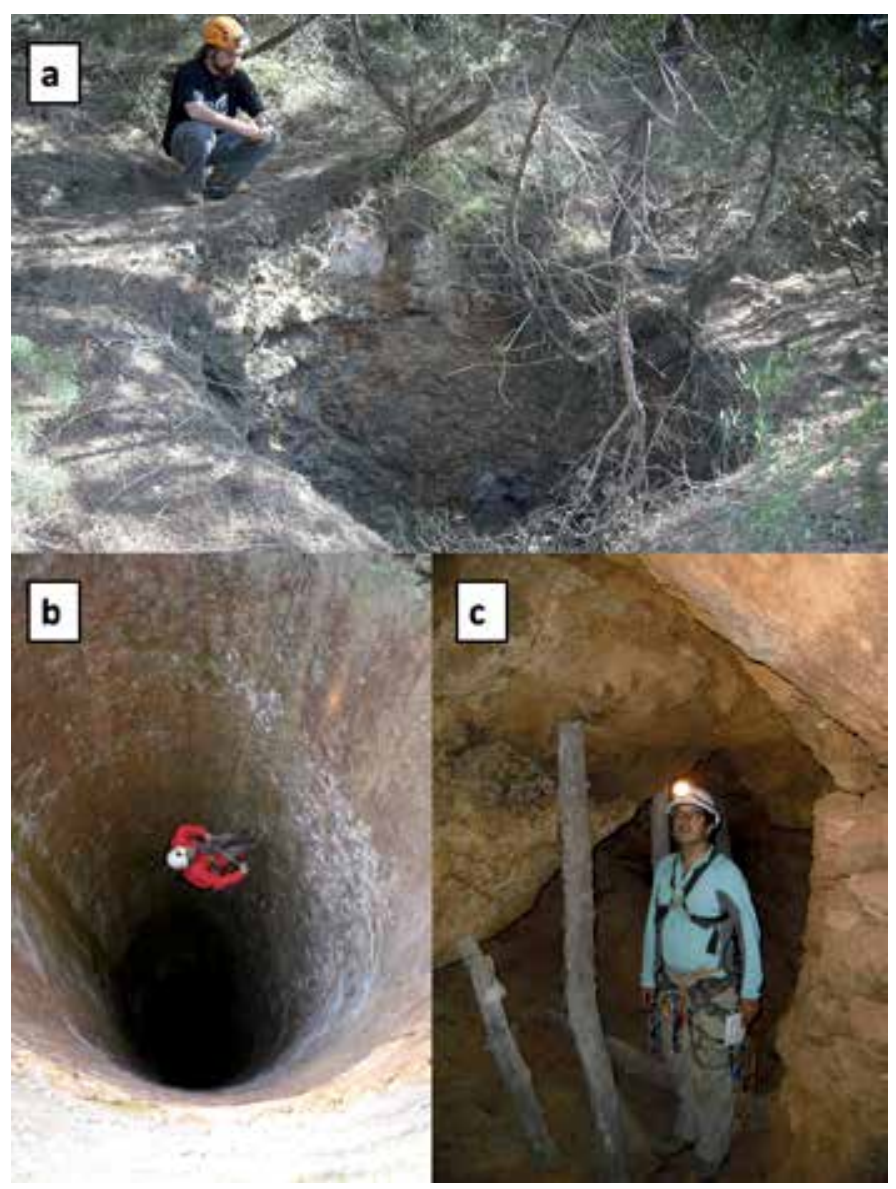

Figura 2. (a) Fotografía mostrando un colapso de un pilar corona de un minado somero en la zona de S'Argentera. Fotografías del interior de las minas de S'Argentera: (b), descenso mediante técnicas espeleológicas a las labores subterráneas, a través de un pozo maestro de 1900; (c) Labores en tajo largo entibadas con madera de sabina y pedriza de relleno, para sujetar zonas antiguas deszafradas. Posibles trabajos de la década de 1860-1870 (Campaña de exploración de junio de 2010).

Figure 2. (a) Photograph showing a crown pillar collapse of a shallow stope in the S'Argentera zone. Photographs showing inside the S'Argentera mines (b) descending to the underground stopes using speleological techniques, through a main shaft dating from 1900 (courtesy Durán Valsero), (c) timbered longwall stopes with sabina tree and rock filling, used to support previous-old recovered mines. Workings dating from 1860-1870 decade (June 2010 expedition). del mismo que ha provocado numerosos colapsos superficiales (Fig.2a)

\section{Metodología}

\section{Aspectos generales}

La metodología del estudio realizado ha comprendido diversos trabajos entre los años 2010 a 2014, siguiendo las pautas habituales internacionalmente aceptadas de análisis del espacio subterráneo (Cornejo y Salvador 1996, Hoek et al 1995, Jordá 2009):

- Reconocimiento geotécnico de campo mediante estaciones geomecánicas del interior de la mina (en este caso particular con ayuda de técnicas espeleológicas - descenso por dos antiguos pozos de mina).

- Evaluación mediante métodos empíricos de estabilidad de las cámaras: índice $\mathrm{Q}$ y método gráfico de estabilidad.

- Evaluación de la estabilidad del pilar corona mediante el "ancho escalado".

- Perfiles de sísmica de refracción (Fig.1 y Fig.3) con objeto de determinar el espesor de suelo - roca alterada e inferir los parámetros geomecánicos Q-Oc del macizo rocoso.

- Modelización numérica y análisis tensodeformacional- aplicando el criterio de Hoek y Brown generalizado (Hoek et al 2002) para macizo rocoso de la sección más desfavorable de la cámara de mayores dimensiones (la oeste).

La evaluación de estabilidad de minas abandonadas debe de contemplar tanto el vano máximo de las galerías o labores en sí en secciones de dos dimensiones (Barton, et.al., 1974, Barton y Bieniawski, 2008, Cornejo y Salvador, 1996), como su interacción con el entorno y las afecciones a la superficie del terreno. En el caso de minados superficiales es crítico el análisis del pilar corona (Carter 2008, 2014, Carter y Miller 1995, Golder 1990). Se denomina pilar corona a la franja de terreno rocoso sin explotar que separa los huecos subterráneos de la superficie y que adecuadamente diseñado evita subsidencias indeseadas y colapsos del terreno: el método escogido para su análisis ha sido el del denominado ancho escalado. La evaluación de la estabilidad de las cámaras debe tener en cuenta además que su forma es claramente tridimensional o poliédrica y el método evaluará cada una de esas caras: por ello tras un primer cálculo del índice Q y su aplicación bidimensional se ha cotejado con un método empírico de análisis de cámaras teniendo en cuenta todas sus caras: que es el llamado método gráfico de estabilidad (Nickson 1992, Potvin 1988, 2014) 
Jordá-Bordehore, et al., 2017. Evaluación de la estabilidad de las labores y pilar... Boletín Geológico y Minero, 128 (1): 3-24

\section{Clasificaciones geomecánicas y análisis empírico. Sistema $Q$}

Los dos sistemas de clasificación geomecánica más empleados son el Rock Mass Rating RMR (Bieniawski 1973, 1989, 2011) y el índice Q (Barton et al, 1974, ). En esta ocasión se empleará el sistema $\mathrm{Q}$ dado que es más apropiado para análisis de vanos variables y porque los otros sistemas a aplicar (Método Gráfico y Ancho Escalado) emplean variaciones de éste.

El Sistema- $\mathrm{Q}$, índice $\mathrm{Q}$ o también llamado “ $\mathrm{Q}$ de Barton", fue desarrollado en Noruega en 1974 por Barton, Lien y Lunde, del Instituto Geotécnico Noruego (Barton et al 1974, NGI, 2014). Se basó su estudio en el análisis de cientos de casos de túneles construidos principalmente en Escandinavia. A lo largo de los últimos 40 años se ha ido generalizando para otros tipos de terreno, actualizando sus parámetros y presentando casos prácticos en túneles y obras subterráneas del mundo entero. Conjuntamente con el Rock Mass Rating o RMR (Barton y Bieniawski 2008) son las clasificaciones geomecánicas internacionalmente más aceptadas.

El índice $\mathrm{Q}$ asigna a cada terreno un valor tanto mayor cuanto mejor es la calidad de la roca. Su variación no es lineal como la del RMR, sino exponencial, y oscila entre $Q=0.001$ para terrenos muy malos $y$ $\mathrm{Q}=1000$ para terrenos muy buenos.

El valor de $\mathrm{Q}$ se obtiene de la siguiente expresión:

$$
Q=\frac{R Q D}{J n} \cdot \frac{J r}{J a} \cdot \frac{J w}{S R F}
$$

Para la descripción pormenorizada y obtención del ROD y cada uno de los cinco últimos parámetros, se remite al lector a la extensa bibliografía sobre el modo de obtener y valorar cada uno de ellos (Barton et.al 1974, NGI 2013)

\section{Estabilidad del pilar corona: método del ancho escalado}

El concepto de ancho escalado para analizar pilares corona es una metodología empírica desarrollada en Canadá en los años 1980 a raíz de varios incidentes producidos por el colapso de pilares corona (Carter, 2014) y está basada en varios cientos de casos reales, tomando los 70 originales del informe de 1990 pero añadiendo nuevos casos mundiales. A lo largo de los últimos 20 años se ha estado empleando en el mundo entero como el método empírico más aceptado para la evaluación preliminar del rango de estabilidad de pilares corona en minados superficiales.
El factor de seguridad para el pilar corona viene de la relación entre el denominado ancho crítico Sc y el ancho escalado Cs, según la fórmula (Carter y Miller 1995):

$$
F O S=\frac{S c}{C s}
$$

El ancho escalado del pilar corona se determina mediante la fórmula (Carter y Miller 1995):

$$
\mathcal{C}_{S}=S\left\{\frac{\gamma}{T\left[\left(1+S_{R}\right)(1-0.4 \cos \theta)\right]}\right\}^{0.5}
$$

\section{Donde:}

Cs $=$ es el ancho escalado en metros.

$\mathrm{S}=$ es el vano real del minado en metros.

$\gamma=$ es la densidad de la roca de la corona en $\mathrm{g} / \mathrm{cm}^{3}$. En caso de sobrecargas, lagunas o material de relleno o tipo suelo se considera como una densidad a añadir o densidad efectiva.

$T=$ es el espesor del pilar corona en metros.

$S_{R}=$ es el ratio entre el ancho o vano y la longitud del minado $=\mathrm{S} / \mathrm{L}$.

$\Theta=$ es el buzamiento del cuerpo mineralizado o de la foliación.

El gráfico original de la Figura 7 (Golder 1990) muestra en abscisas la calidad de la roca según el índice $\mathrm{Q}$ o $\mathrm{RMR}_{76}$ (que se diferencia del posterior del 79-89 en la evaluación de las calidad de discontinuidades) y en ordenadas el ancho escalado (Cs). En este gráfico se han ido situando los puntos correspondientes a los minados analizados y que forman el histórico del método empírico: con color negro encontramos los minados hundidos o en estado muy precario (caving) y en hueco o blanco encontramos los minados estables- entre ambas zonas se pueden trazar varias posibles líneas límite y que corresponden a varias formulaciones del denominado ancho crítico (Sc).

Los resultados de la estabilidad del pilar corona pueden, además de evaluarse según el factor de seguridad, introducir la probabilidad de fallo del mismo, considerando las incertidumbres por la variabilidad de la calidad $\mathrm{Q}$, el buzamiento de estratos, geometría de la cámara, etc. La fórmula de probabilidad de fallo en función del factor de seguridad es según Carter (2014):

$$
P f(\%)=\frac{100}{1+441 \times e^{\left(-1,7 C s / Q^{0,44}\right)}}
$$


Una vez analizado el pilar corona desde el punto de vista de la seguridad (FoS) y de su probabilidad de fallo (Pf\%) resulta de sumo interés de cara a las autoridades, clasificar los rangos de estabilidad y en base a ello establecer unas recomendaciones sobre accesibilidad o prohibición de acercamiento a esas zonas, los cuales tienen implicaciones en las medidas a tomar: vallado de un perímetro, auscultación, relleno de labores 0 , por ejemplo, voladura del pilar corona (tabla 4).

\section{Método gráfico de estabilidad}

Dado que las cámaras son huecos tridimensionales su análisis debe de realizarse preferentemente como una estructura poliédrica, para ello se emplea el método gráfico de estabilidad, más apropiado que las clasificaciones geomecánicas directas RMR y $\mathrm{Q}$ las cuales son más empleadas para pre dimensionar obras longitudinales como son los túneles y galerías.

El método gráfico de estabilidad es una metodología ampliamente usada en el proceso de prediseño de caserones o cámaras mineras de grandes dimensiones. Se emplea para definir las dimensiones de unidades de explotación de cuerpos tabulares, normalmente verticalizados (Potvin 1988).). El procedimiento de diseño se basa en el cálculo de dos factores, que se llevan a un gráfico como abscisas y ordenadas, el cual está dividido empíricamente en sectores (Fig.10):

- Número de estabilidad modificado. N'. Basado en el índice $\mathrm{Q}$, representa la habilidad del macizo rocoso para permanecer estable bajo unas determinadas condiciones de esfuerzos.

- Radio hidráulico. S. Es el factor de forma, que tiene en cuenta la forma y tamaño de la pared o techo de la cámara de la superficie que se esté estudiando y se determina como: S = Área de la sección a analizar / perímetro de la superficie.

El número de estabilidad $\mathrm{N}^{\prime}$, está basado en el índice de calidad $Q$, aunque no directamente sino a través del $Q^{\prime}$, siendo éste:

$$
\begin{aligned}
& N^{\prime}=Q^{\prime} \times A \times B \times C \\
& Q^{\prime}=\frac{R Q D}{J n} \times \frac{J r}{J a} \times \frac{1}{1}
\end{aligned}
$$

Dónde:

$\mathrm{Q}^{\prime}$ = es el índice de calidad $\mathrm{Q}$ modificado, fijando el factor $S R F=1 ., y$ dado que no se basa en casos con importantes cantidades de agua, o bien son minados drenados o sin presión hidrostática, se emplea $\mathrm{Jw}=1$.

La evaluación de cada uno de estos factores de corrección A, B, C está ampliamente descrita en la literatura (Potvin 1988, 2014, Hoek et al 1995).

\section{Modelo tensodeformacional - criterio de Hoek y Brown}

Para modelizar el comportamiento tensodeformacional de las cámaras de las minas y terreno circundante se ha empleado el software Phase 2 v9 de la casa Rocscience que trabaja mediante elementos finitos en dos dimensiones y se ha adoptado el criterio de rotura de Hoek -Brown generalizado . La fórmula siguiente es la ecuación del criterio generalizado de Hoek y Brown (Hoek et al 2002, Hoek, 2007):

$\sigma^{\prime} 1=\sigma^{\prime} 3+\sigma c i\left(m b \times \frac{\sigma^{\prime} 3}{\sigma c i}+s\right)^{a}$

Los parámetros de la Ecuación 7 se pueden estimar a partir de ensayos de laboratorio y de campo: triaxiales y compresión simple para la matriz rocosa y de caracterizaciones de campo para evaluar el macizo rocoso en su conjunto (Hoek, 2007)

$$
\begin{aligned}
& m b=m i \times e^{\left(\frac{G S I-100}{28-14 D}\right)} \\
& s=e^{\left(\frac{G S I-100}{9-3 D}\right)} \\
& a=\frac{1}{2}+\frac{1}{6}\left(e^{\frac{-G S I}{15}}-e^{\frac{-20}{3}}\right)
\end{aligned}
$$

\section{Resultados}

\section{Evaluación de la estabilidad mediante índice $Q$ de Barton}

Se ha determinado el valor del índice $\mathrm{Q}$ por separado para las cámaras oeste, utilizando las estaciones geomecánicas EG\#4 y EG\#5 y la cámara este con la EG\#6 (tabla 1) 


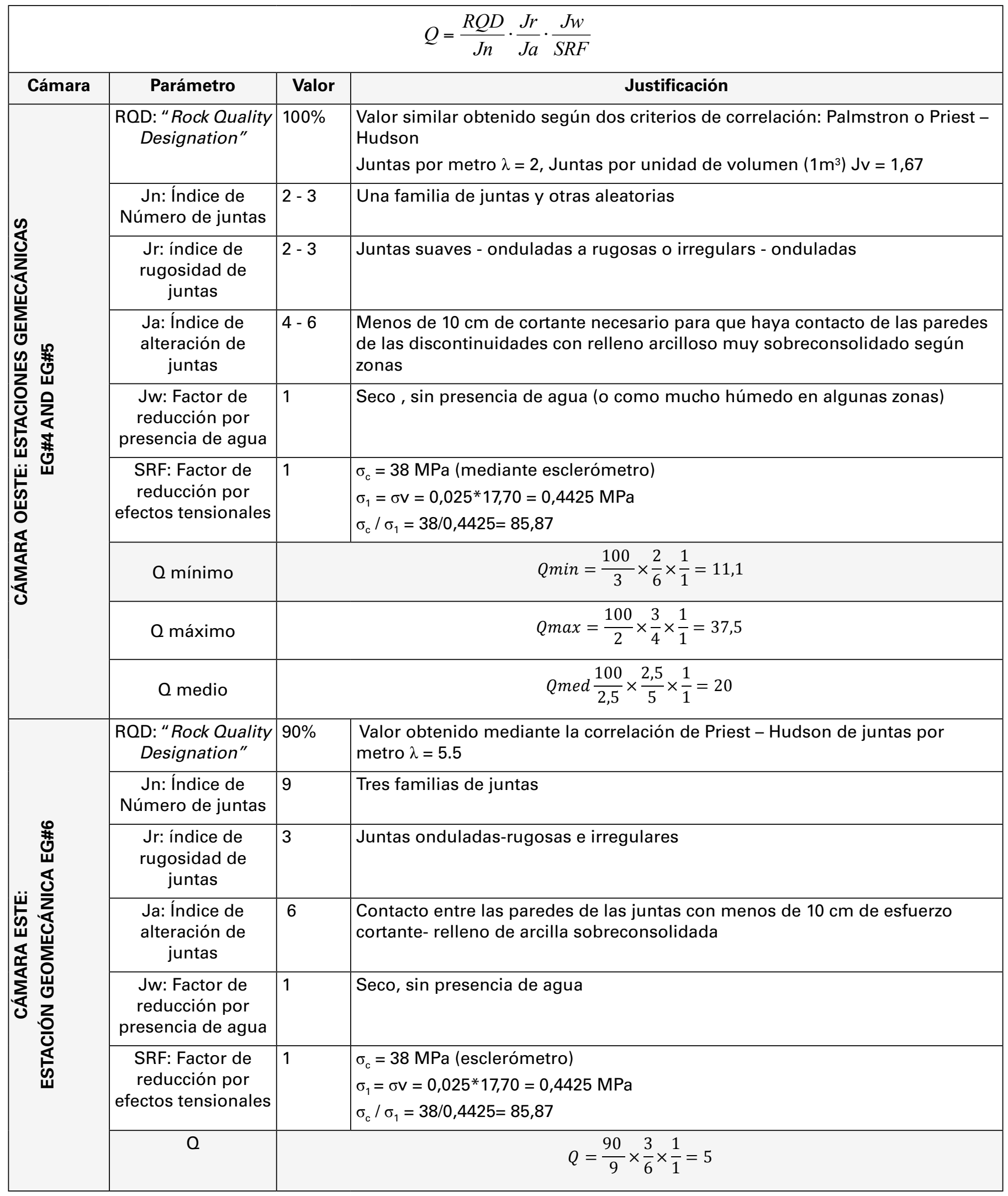

Tabla 1. Determinación del valor del índice $Q$ en las cámaras oeste y este a partir de las estaciones geomecánicas E\#4, EG\#5 y EG\#6. Table 1. Determination of the $Q$ index in the west and east stopes using the geomechanical stations EG\#4, EG\#5 and EG\#6. 

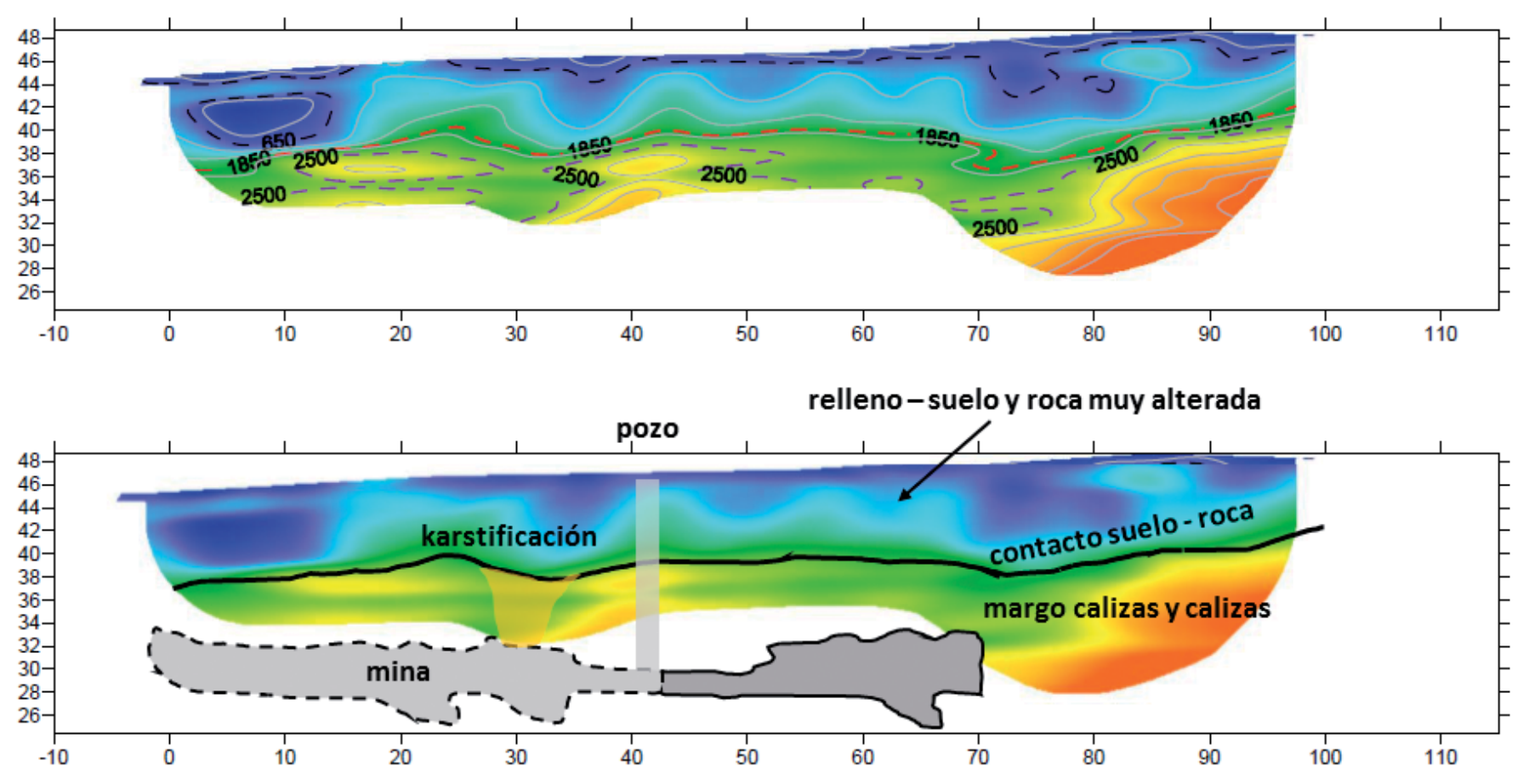

Perfil sísmica refracción PS-1

Figura 3. Perfil de sísmica de refracción (PS\#1) sobre la cámara minera este y próximo a la cámara oeste. Debajo se muestra la interpretación geológica.

Figure 3. Seismic refraction survey (PS\#1) above the east mining stope and nearby the west stope. Below the geological interpretation is shown.

En la mayoría de las ocasiones no es factible por motivos de seguridad, el acceso a las labores mineras abandonadas para realizar estaciones geomecánicas, en cuyo caso las técnicas indirectas geofísicas son fiables para determinar la forma de la interfase suelo-roca y la competencia del macizo rocoso en profundidad. En este caso particular, se ha empleado la sísmica de refracción para cotejar los valores de calidad del macizo rocoso y para determinar el contacto suelo roca y que delimita el espesor real del pilar corona a $7 \mathrm{~m}$. (Fig.3). Para correlacionar la Vp (en $\mathrm{m} / \mathrm{s}$ ) con la calidad de roca Qc (el parámetro Qc es el $\mathrm{O}$ multiplicado por el cociente $\sigma \mathrm{c} / 100)$ se emplea el gráfico de la Figura 5 (original de Barton, 2002). Para la adquisición sísmica se ha empleado un sismógrafo Geometrics de 24 canales y un espaciado de geófonos de $5 \mathrm{~m}$ y un total de 6 a 7 disparos.

En la zona del punto 70 - 80 (Fig.3) se aprecia como la velocidad decrece generando una falsa cubeta dado que la interfase suelo roca sigue siendo sensiblemente horizontal. Esta anomalía es debida a la presencia de grandes huecos que generan una anomalía de baja velocidad de $1800 \mathrm{~m} / \mathrm{s}$ en vez de los $2500 \mathrm{~m} / \mathrm{s}$ o más del macizo encajante.

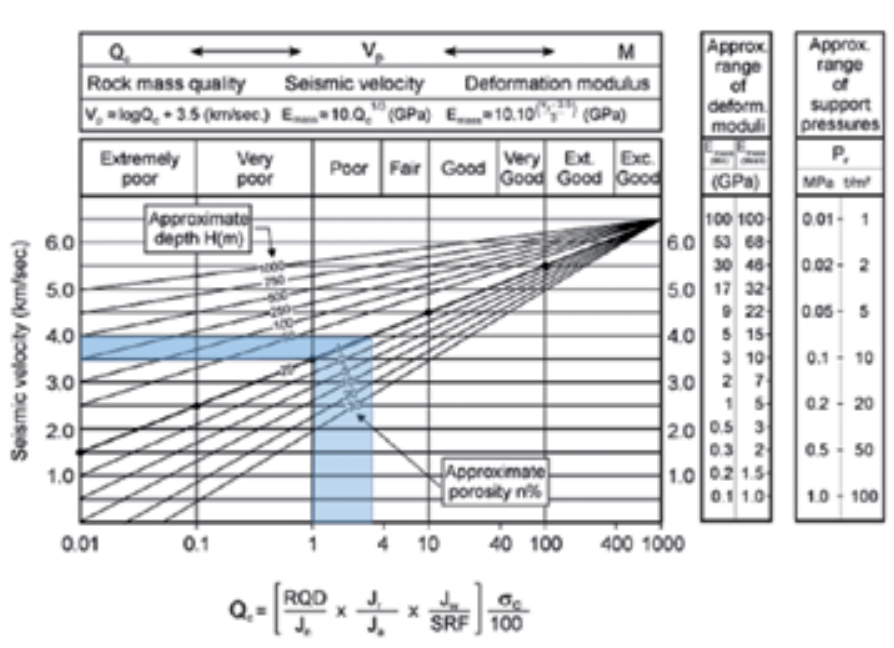

Figura 4. Relación entre la velocidad sísmica $\mathrm{Vp}(\mathrm{m} / \mathrm{s})$ y la calidad del macizo rocoso Qc, con los rangos que se han medido en el estudio (modificado sobre gráfico original de Barton, 2002).

Figure 4. Relation between seismic velocity $V p(\mathrm{~m} / \mathrm{s})$ and rock mass quaility $Q c$, with the ranges measured in the study (modified over the original graph by Barton, 2002) 
En ordenadas (Fig.4) se ha situado el rango de velocidades sísmicas que se asumen razonables para el macizo rocoso de las minas $(3400 \mathrm{~m} / \mathrm{s}<\mathrm{Vp}<4000 \mathrm{~m} / \mathrm{s})$, tomando los datos del perfil (Fig.4). Intersectando la recta de cota $25 \mathrm{~m}$, obtenemos una $\mathrm{Qc}$ variable entre $1-3,5$ que equivaldría a una $O$ de 2,6 - 9,2 considerando un ratio $\sigma c / 100=38 / 100$. Estos valores son coherentes con los obtenidos en el mapeo directo subterráneo mediante estaciones geomecánicas $(5<0<37,5)$.

El gráfico de sostenimiento de la Figura 5 es el más aceptado internacionalmente para prediseño de sostenimientos en una galería, túnel o caverna mediante la calidad de la roca - índice $\mathrm{Q}$ y el vano máximo. El ancho real de la cámara se "mayora" a efectos de diseño mediante un factor de seguridad denominado "Excavation Support Ratio" -ESR, que en el caso de una cavidad permanente de acceso por personal podría estar en 0,8 . La zona situada hacia la esquina inferior derecha (Fig. 5) corresponde a excelente calidad de roca y vanos relativamente pequeños, lo que no requiere sostenimiento. La cámara oeste es actualmente estable, tal y como queda corroborado por el gráfico (Fig. 5) si se introduce el vano real sin mayorar, el punto se ubica en la zona de estable sin soporte; sin embargo si se tiene en cuenta el ESR penalizador (como debe de ser para una zona visitable) resulta que para valores mínimos y medios de $\mathrm{Q}$ se requiere algo de sostenimiento (p.e. bulones para las cuñas). Se observa que la cámara principal, tras más de 100 años desde su abandono total se ha estabilizado totalmente y presenta sólo algunos bloques rocosos puntuales desprendidos o inestables de mucho tiempo atrás, prácticamente coetáneos con el abandono de la mina. La cámara este está ubicada en la zona del gráfico (punto 3 Fig.5) donde se recomienda refuerzo.

Se aplica el factor ESR a los diferentes vanos y alturas posibles, considerando un posible uso turístico a la cavidad; se propone un $E S R=0,8$.

Para la cámara oeste: Vano / ESR $=6 / 0,8=7,5 \mathrm{~m}$.

Para la cámara este: Vano $/ E S R=4,5 / 0,8=5,6 \mathrm{~m}$.

Para la altura de ambas cámaras: Altura / ESR $=5$ / 0,8 $=6,25 \mathrm{~m}$.

\section{Estabilidad del pilar corona}

En el caso de las minas abandonadas de S'Argentera se ha evaluado la estabilidad del pilar corona del hueco con mayores dimensiones: el que contempla dos cámaras, la Este y la Oeste, esta última además pasa por debajo de una carretera (Fig.1). En el análisis no todo el terreno hasta la superficie

\begin{tabular}{|c|c|c|}
\hline \multicolumn{2}{|r|}{ Tipo de excavación } & ESR \\
\hline$A$ & Labores mineras temporales, etc. & ca. 3-5 \\
\hline B & $\begin{array}{l}\text { Pozos verticales*: } \\
\text { i. Sección circular } \\
\text { ii. Sección rectangular o cuadrada } \\
\left({ }^{*}\right) \text { dependiendo de la función, pueden ser } \\
\text { menores que los valores dados }\end{array}$ & $\begin{array}{l}c a .2 .5 \\
c a .2 .0\end{array}$ \\
\hline C & $\begin{array}{l}\text { Huecos mineros permanentes, túneles } \\
\text { de centrales hidroeléctricas (excluyendo } \\
\text { las galerías de alta presión), túneles } \\
\text { de suministro de agua, túneles } \\
\text { piloto, galerías de avance en grandes } \\
\text { excavaciones. }\end{array}$ & 1.6 \\
\hline $\mathrm{D}$ & $\begin{array}{l}\text { Túneles menores de carretera y ferrocarril, } \\
\text { chimeneas de equilibrio, túneles de } \\
\text { acceso, colectores, etc... }\end{array}$ & 1.3 \\
\hline$E$ & 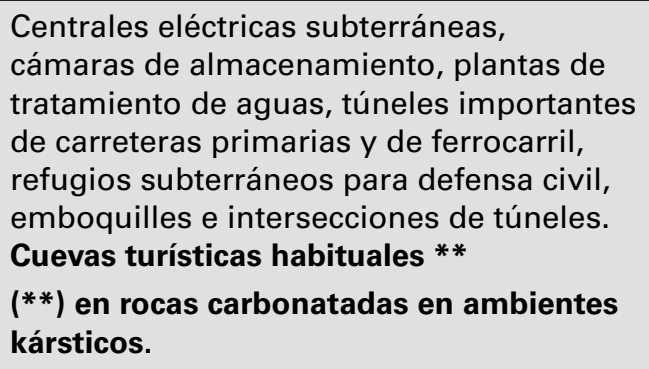 & 1.0 \\
\hline $\mathrm{F}$ & $\begin{array}{l}\text { Centrales nucleares subterráneas, } \\
\text { estaciones de ferrocarril, instalaciones } \\
\text { públicas y deportivas, fábricas, etc. } \\
\text { Minas turísticas, entradas y bocaminas } \\
\text { en minas y cuevas turísticas, rutas bajo } \\
\text { vanos de cuevas no kársticas, tubos } \\
\text { de lava turísticos, cuevas y viviendas } \\
\text { trogloditas hechas por el hombre. }\end{array}$ & 0.8 \\
\hline G & $\begin{array}{l}\text { Cavernas muy importantes y huecos } \\
\text { subterráneos con una duración de vida } \\
\text { larga, } 100 \text { años o sin acceso para } \\
\text { mantenimiento. } \\
\text { Grandes vanos y minas y cuevas } \\
\text { turísticas importantes con espectáculos } \\
\text { subterráneos, auditorios, restaurantes, } \\
\text { capillas, etc. }\end{array}$ & 0.5 \\
\hline
\end{tabular}

Tabla 2. Valores propuestos de ESR para las cuevas y minas turísticas, añadidos como subcategorías al gráfico original (modificado de NGI, 2013).

Table 2. Proposed ESR values for show caves and mines, added as sub-categories to the original chart (modified from NGI, 2013). 


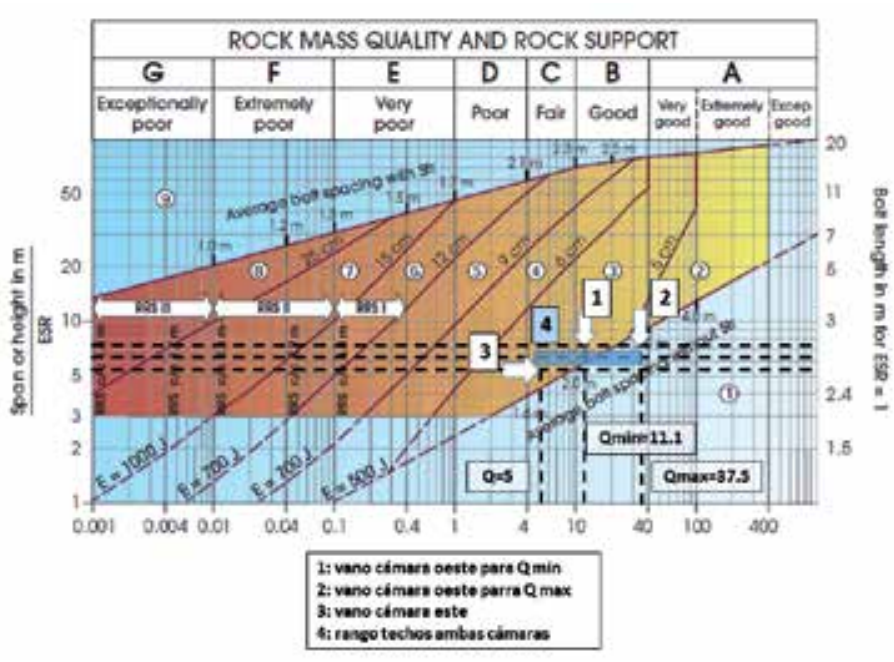

Figura 5. Gráfico de sostenimientos recomendados según índice $\mathrm{Q}$ aplicado a las cámaras principales oeste y este de la mina de S'Argentera (modificado de NGI, 2013).

Figure 5. Chart of recommended reinforcement base on $Q$, applied to the main stopes west and east in the S'Argentera mine (Modify from NGI, 3013).

corresponde a "pilar corona", es decir, es macizo rocosopero una parte superficial de $6 \mathrm{~m}$ corresponde a suelo o material de relleno y el pilar tiene un espesor de unos $7 \mathrm{~m}$ - de los $13 \mathrm{~m}$ totales hasta la superficie. El espesor de esta cobertera se ha determinado mediante los perfiles de sísmica de refracción.

Determinación de la densidad efectiva en espesor de pilares corona: En el caso que se analiza el pilar corona tiene un espesor medio de $7 \mathrm{~m}$ y una a

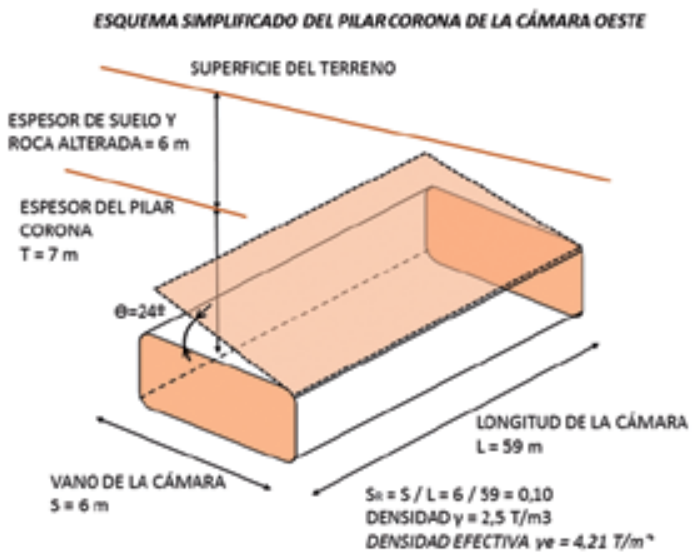

b

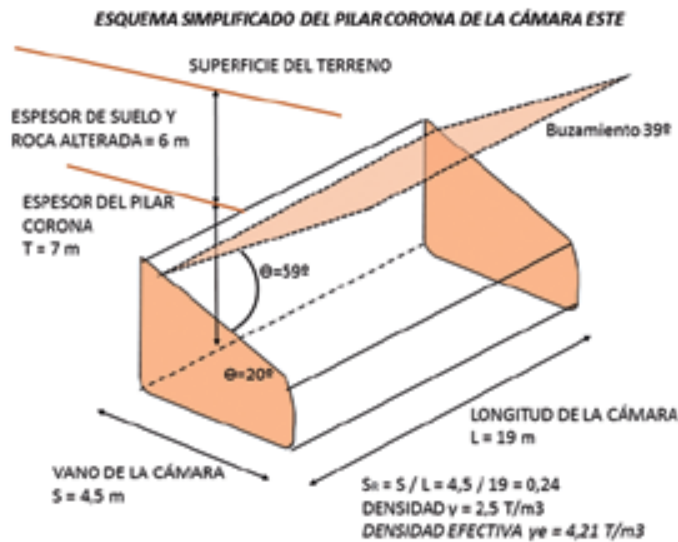

Figura 6. Esquema simplificado para análisis de los pilares corona de las cámaras oeste $(A)$ y este $(B)$.

Figure 6. Simplified scheme of the west $(A)$ and east $(B)$ mine stopes considered for the scaled span assessment.

\begin{tabular}{|l|c|c|c|}
\hline & Fórmula & Valor cámara Oeste & Valor cámara Este \\
\hline Índice Q & $Q=\frac{R Q D}{J n} \cdot \frac{J r}{J a} \cdot \frac{J w}{S R F}$ & $Q \min =\frac{100}{3} \times \frac{2}{6} \times \frac{1}{1}=11,1$ & $Q=\frac{90}{9} \times \frac{3}{6} \times \frac{1}{1}=5$ \\
\hline $\begin{array}{l}\text { Ancho critico Sc } \\
\text { (Golder 2008) }\end{array}$ & $S c=3,58 \times Q^{0,44}$ & $S c=3,58 \times(11,1)^{0,44}=10,31 \mathrm{~m}$ & $\mathrm{Sc}=3,58 \times(5)^{0,44}=2,03 \mathrm{~m}$ \\
\hline $\begin{array}{l}\text { Ancho escalado Cs } \\
\text { (Golder 1990) }\end{array}$ & $C s=\left[\frac{\gamma}{T(1+S R)(1-0,4 \cos \emptyset)}\right]^{0,5}$ & $C s=\left[\frac{4,21}{7(1+0,10)(1-0,4 \cos 24)}\right]^{0,5}$ & $C s=\left[\frac{4,21}{7(1+0,24)(1-0,4 \cos 59)}\right]^{0,5}=0,78 \mathrm{~m}$ \\
\hline $\begin{array}{l}\text { Factor de seguridad } \\
\text { (Golder 1990) }\end{array}$ & $F o S=\frac{S c}{C s}$ & $F o S=\frac{10,31}{0,93}=11,09$ & $F o S=\frac{2,03}{0,78}=2,6$ \\
\hline $\begin{array}{l}\text { Probabilidad de fallo } \\
\text { Pf(\%) (Cottrell et.al } \\
\text { 2009) }\end{array}$ & $P f(\%)=\frac{100}{1+441 \times e^{\left(-1,7 C s / Q^{0,44}\right)}}$ & $P f(\%)=\frac{100}{255,78}=0,39 \%$ & $P f(\%)=\frac{100}{847,72}=0,12 \%$ \\
\hline
\end{tabular}

Tabla 3. Parámetros básicos en el ancho escalado. Análisis de ancho escalado, ancho crítico, factor de seguridad y probabilidad de fallo para ambas cámaras.

Table 3. Basic parameters for the scaled span. Assessment of the scald span, critical span, factor of safety and probability of failure for both stopes. 
sobrecarga de suelo de $6 \mathrm{~m}$. Considerando una densidad para la roca de $2,5 \mathrm{t} / \mathrm{m}^{3}$ y para el suelo de $2,0 \mathrm{t} /$ $\mathrm{m}^{3}$, la densidad efectiva mayorada sería

$$
\gamma e f f=\frac{(2,0 \times 6+2,5 \times 7)}{7}=4,21 t / m 3
$$

Para obtener el vano crítico (Sc) se considera un valor de $\mathrm{Q}$ conservador en ambas cámaras: el valor mínimo de $\mathrm{Q}=11,1$ para la Oeste y el único valor de $\mathrm{Q}=5$ para la Este (Tabla 3 ).

La evaluación de la estabilidad del pilar corona por el método del ancho escalado (Tabla 2) arroja un factor de seguridad (FoS) mayor de 2 para ambas cámaras y una probabilidad de fallo también en ambos casos menor de 0,5 , por tanto ambas cámaras y pilares corona se ubicarían en la categoría $\mathrm{G}$ donde se puede permitir libremente el acceso.

Además de evaluar la estabilidad según FoS y Probabilidad (Tabla 4) se pueden representar directamente los valores de calidad y ancho escalado en el gráfico actualizado (Fig.7) y comprobar cuán lejos o cerca se está de la línea crítica (Sc). Además de los valores de $\mathrm{Q}$ representados en abscisas del mapeo geomecánico, se ha representado el valor de GSI $=67$ determinado visualmente en la cámara grande y que correlaciona perfectamente con $\mathrm{Q}$, así como el rango de $Q$ determinado a partir de $Q c$ y $V p(\mathrm{~m} / \mathrm{s})$.

\begin{tabular}{|c|c|c|c|c|c|c|c|c|}
\hline \multirow{3}{*}{$\begin{array}{c}\text { Clase } \\
\\
\text { A }\end{array}$} & \multirow{3}{*}{$\begin{array}{c}\begin{array}{c}\text { Probabilidad } \\
\text { de fallo }(\%)\end{array} \\
50-100\end{array}$} & \multirow{3}{*}{$\begin{array}{c}\begin{array}{c}\text { Fiabilidad } \\
(\%)\end{array} \\
0-50\end{array}$} & \multirow{3}{*}{$\begin{array}{c}\begin{array}{c}\text { Factor de } \\
\text { seguridad } \\
\text { mínimo }\end{array} \\
<1,0\end{array}$} & \multicolumn{5}{|c|}{ Criterios de diseño para una probabilidad de fallo aceptable } \\
\hline & & & & \multicolumn{2}{|c|}{$\begin{array}{l}\text { Vida de servicio del pilar } \\
\text { corona (años) }\end{array}$} & \multirow{2}{*}{$\begin{array}{l}\begin{array}{l}\text { Acceso } \\
\text { público }\end{array} \\
\text { Prohibido }\end{array}$} & \multirow{2}{*}{$\begin{array}{c}\text { Actitud } \\
\text { reguladora ante } \\
\text { el problema } \\
\begin{array}{c}\text { Totalmente } \\
\text { inaceptable }\end{array}\end{array}$} & \multirow{2}{*}{$\begin{array}{c}\begin{array}{c}\text { Supervisión } \\
\text { requerida }\end{array} \\
\text { No es efectivo }\end{array}$} \\
\hline & & & & $\begin{array}{l}\text { Cero en términos } \\
\text { efectivos }\end{array}$ & $<0,5$ & & & \\
\hline B & $20-50$ & $50-80$ & 1,0 & $\begin{array}{l}\text { Muy corto plazo } \\
\text {-solo para propósitos } \\
\text { mineros temporales - } \\
\text { riesgo inaceptable en } \\
\text { emboquille de túneles }\end{array}$ & 1,0 & $\begin{array}{l}\text { Fuertemente } \\
\text { evitado }\end{array}$ & No aceptable & $\begin{array}{l}\text { Monitoreo } \\
\text { continúo } \\
\text { sofisticado }\end{array}$ \\
\hline $\mathrm{C}$ & $10-20$ & $80-90$ & 1,2 & $\begin{array}{l}\text { Muy corto plazo - } \\
\text { pilares corona casi } \\
\text { temporales - riesgo } \\
\text { no deseable en } \\
\text { trabajos temporales } \\
\text { de ingeniería civil }\end{array}$ & $2-5$ & $\begin{array}{l}\text { Activamente } \\
\text { evitado }\end{array}$ & $\begin{array}{l}\text { Muy } \\
\text { preocupante }\end{array}$ & $\begin{array}{l}\text { Monitoreo } \\
\text { continuo con } \\
\text { instrumentos }\end{array}$ \\
\hline $\mathrm{D}$ & $5-10$ & $90-95$ & 1,5 & $\begin{array}{l}\text { Corto plazo - coronas } \\
\text { semi temporales, } \\
\text { por ejemplo debajo } \\
\text { de infraestructuras } \\
\text { mineras poco } \\
\text { sensibles }\end{array}$ & $5-10$ & Evitado & preocupante & $\begin{array}{l}\text { Monitoreo } \\
\text { continuo } \\
\text { simple }\end{array}$ \\
\hline $\mathrm{E}$ & $1,5-5$ & $95-98.5$ & 1,8 & $\begin{array}{l}\text { Medio plazo - coronas } \\
\text { semi permanentes } \\
\text { posibilidad bajo } \\
\text { infraestructuras }\end{array}$ & $15-20$ & Disuadido & $\begin{array}{c}\text { Algo } \\
\text { preocupante }\end{array}$ & $\begin{array}{l}\text { Monitoreo } \\
\text { superficial }\end{array}$ \\
\hline $\mathrm{F}$ & $0,5-1.5$ & $98,5-99.5$ & 2 & $\begin{array}{l}\text { Lago plazo- coronas } \\
\text { casi permanentes - } \\
\text { portales de obra civil - } \\
\text { túneles alcantarillado }\end{array}$ & $50-100$ & Permitido & $\begin{array}{l}\text { Preocupación } \\
\text { limitada }\end{array}$ & $\begin{array}{l}\text { Monitoreo } \\
\text { superficial } \\
\text { ocasional }\end{array}$ \\
\hline
\end{tabular}

Tabla 4. Categorías de estabilidad, consecuencias y actuaciones según resultados del análisis por ancho escalado se destacan los casos del presente trabajo (traducido y modificado de Carter, 2014).

Table 4. Categories of stability, consequences and actions based on the results of the scaled span analysis, highlighted those form this study (translated and modified from Carter, 2014). 


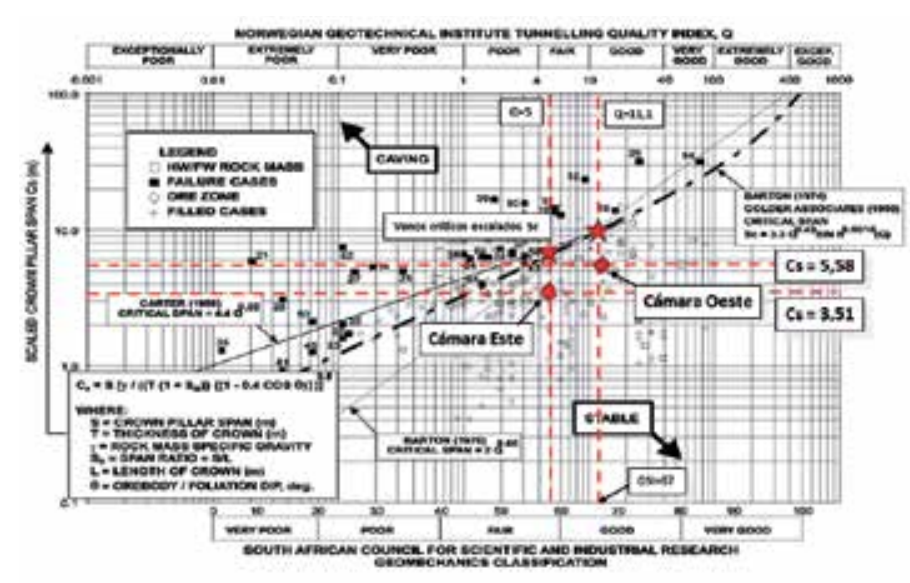

Figura 7. Estabilidad de pilar corona para los datos específicos de S'Argentera sobre el grafico original de estabilidad según los casos analizados en la década de los 1980's (modificado de Golder, 1990). Figure 7. Crown pillar stability based on S'Argentera specific data, over the original stability graph according to the case studies of the decade of the 1980's (modified form Golder, 1990).

\section{Resultados método gráfico de estabilidad}

La estabilidad debe determinarse para cada una de las caras que conforman el poliedro de la cámara que se está evaluando, es decir: techo, hastial a techo y hastial a muro.

Los valores del índice $\mathrm{Q}^{\prime}$ considerados son los siguientes:

Para la cámara oeste se toma el Q' mínimo, para estar del lado de la seguridad

$$
Q^{\prime}(\text { cámara Oeste })=\frac{100}{3} \times \frac{2}{6}=11,1
$$

En el caso de la cámara Este sólo hay un valor del índice $Q^{\prime}$ :

$$
Q^{\prime}(\text { cámara Este })=\frac{90}{9} \times \frac{3}{6}=5
$$
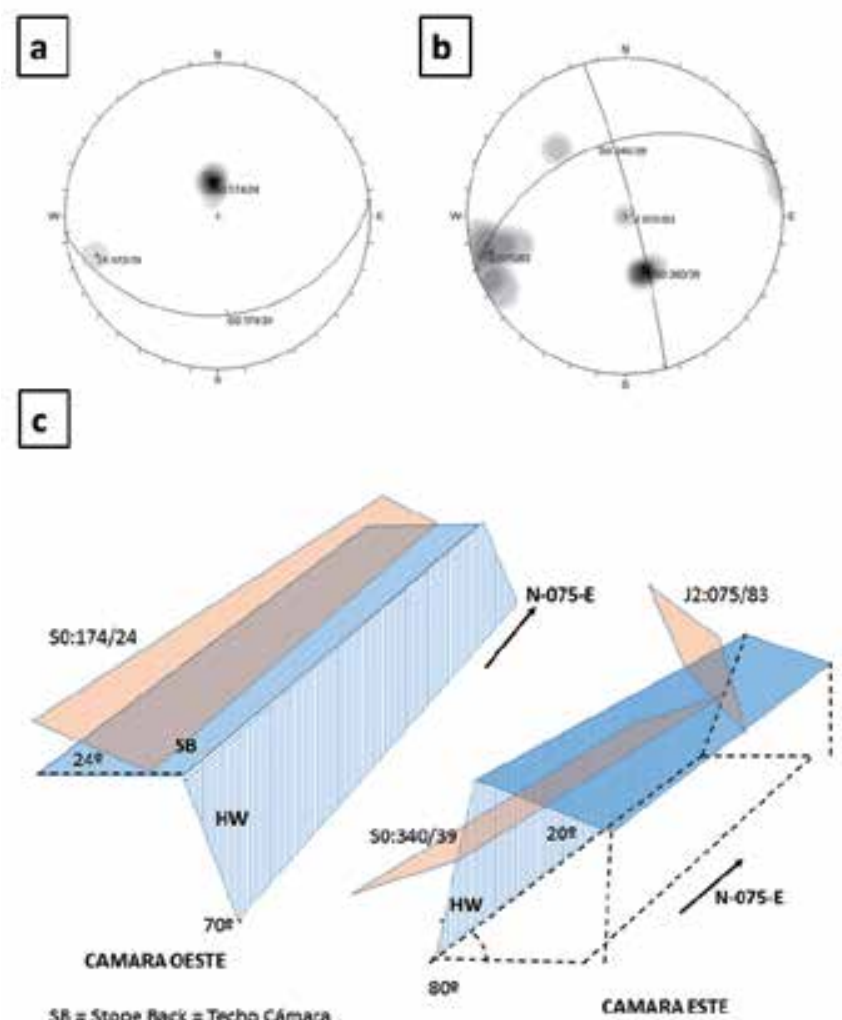

$H W=$ Hanging Wall $=$ Hastial a tech

Figura 8 (a) Representación estereográfica de las familias de discontinuidades en la cámara oeste (empleando software DIPSv5). (b) Representación estereográfica de las familias de discontinuidades en la cámara este (empleando software DIPSv5). (c) Geometría básica de las cámaras utilizada para el análisis de estabilidad (para el método gráfico)

Figure 8. (a). Stereographic representation of the discontinuity sets of the west stope (using DIPSv5 software). (b) Stereographic representation of the discontinuity sets of the east stope (using DIPSV5 software). (c ) Basic geometry of the stopes used for testability assessment.

Las Figura 9 muestra los gráficos para determinar los valores de los parámetros $A, B, C$ particularizados para las cámaras de S'Argentera y la tabla 5 las orientaciones de las familias de juntas consideradas.

\begin{tabular}{|l|l|c|c|}
\hline \multicolumn{1}{|c|}{ Cámara } & \multicolumn{1}{|c|}{ Discontinuidad } & $\begin{array}{c}\text { Direccion de buzamiento } \\
\text { (Dip Direction) en grados }\end{array}$ & Buzamiento (Dip) en grados \\
\hline \multirow{2}{*}{ Oeste } & Estratificación S0 & 174 & 24 \\
\cline { 2 - 4 } & Junta J1 & 072 & 79 \\
\hline \multirow{2}{*}{ Este } & Estratificación S0 & 340 & 39 \\
\cline { 2 - 4 } & Junta J & 075 & 83 \\
\hline
\end{tabular}

Tabla 5. Orientaciones de las principales familias de discontinuidades en cada una de las cámaras.

Table 5. Orientation of main joint sets in each stope. 


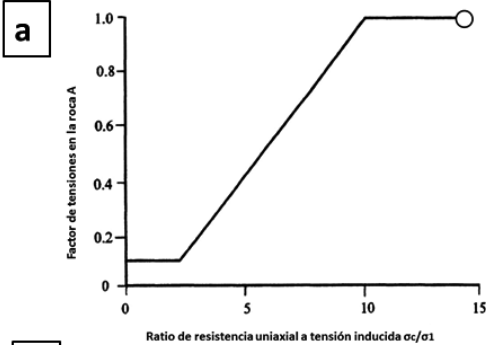

C
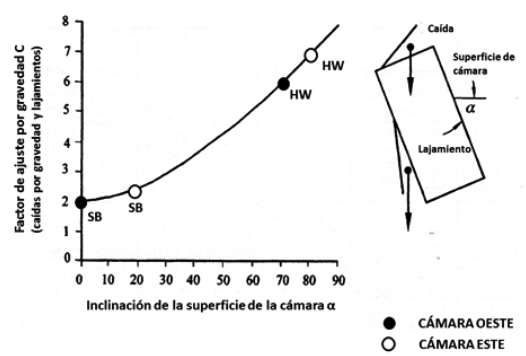
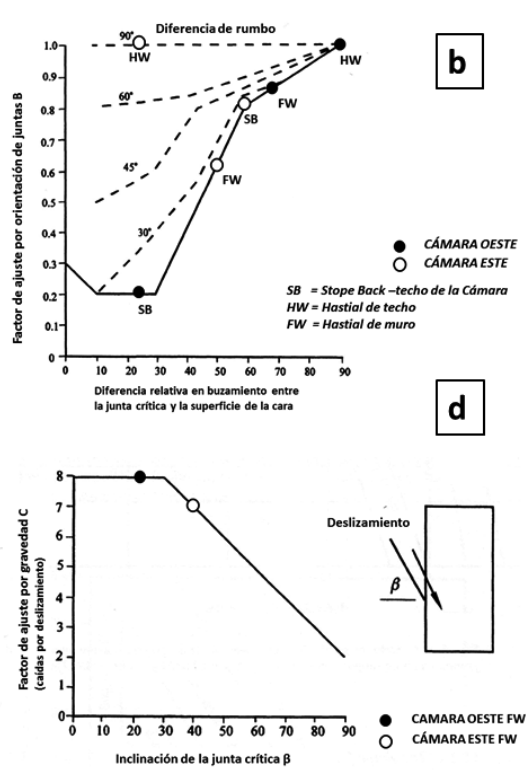

Figura 9. Determinación de los parámetros del método gráfico de estabilidad para el caso particular de las cámaras mineras de S'Argentera (A) Factor de tensiones en la roca, para diferentes valores de $\sigma_{\mathrm{c}} / \sigma_{1}$ (modificado de Potvin 1988). (C) Factor de ajuste B, que tiene en cuenta la orientación de la junta con respecto a la superficie de la cámara (modificado de Potvin 1988) (D) Factor de ajuste de gravedad $C$ para caídas por gravedad y lajamientos (Potvin 1988). (E) Factor de ajuste de gravedad C para roturas por deslizamiento (Potvin 1988), se emplea únicamente para hastiales de techo.

Figure 9. Stability graph parameter determination for the particular case of the S'Argentera mined stopes (A). Rock Stress factor A for different values of $\sigma_{d} / \sigma_{1}$ (modified from Potvin 1988). (B) Critical joint orientation in the stope back with respect to the opening Surface (modified form Potvin, 1988). This parameter applies often to the hanginwall rather than the stope back, when the back is flat, there is no difference in strike. (C) Adjustement factor B, accounting for the orientation of the joint with respect to the stope Surface (modified from Potvin, 1988). (D). Gravity adjustment factor $C$ for gravity falls and slabbing (Potvin, 1988). (E) Gravity adjustment factor $C$ for sliding failure modes (Potvin, 1988) only applied for hangingwall.

\begin{tabular}{|c|c|c|c|c|c|c|}
\hline Cámara & Cara & $\mathbf{Q}^{\prime}$ & A & B & C & $\mathbf{N}^{\prime}$ \\
\hline \multirow{3}{*}{ Oeste } & SB & 11.1 & 1 & 0.2 & 2 & 4.4 \\
\cline { 2 - 7 } & HW & 11.1 & 1 & 1 & 6 & 66.6 \\
\cline { 2 - 7 } & FW & 11.1 & 1 & 0.85 & 8 & 75.5 \\
\hline \multirow{3}{*}{ Este } & SB & 5 & 1 & 0.80 & 2.5 & 10 \\
\cline { 2 - 7 } & HW & 5 & 1 & 1 & 7 & 35 \\
\cline { 2 - 7 } & FW & 5 & 1 & 0.60 & 7 & 21 \\
\hline
\end{tabular}

Tabla 6. Resumen de los factores de corrección y determinación del número de estabilidad N’ para cada una de las caras de las cámaras oeste y este de S'Argentera

Table 6. Summary of the correction factors and determination of the stability number N'for earch of the stopes West and East of S'Argentera.

\begin{tabular}{|c|c|c|c|c|c|c|}
\hline Cámara & Cara & $\begin{array}{l}\text { Lado } X \\
\text { en } \mathbf{m}\end{array}$ & $\begin{array}{l}\text { Lado Y en } \\
\mathbf{m}\end{array}$ & $\begin{array}{c}\text { Área }=\mathbf{X Y} \text { en } \\
\mathbf{m}^{2}\end{array}$ & $\begin{array}{c}\text { Perímetro }=2(X+Y) \\
\text { en } m\end{array}$ & $\begin{array}{c}\text { Radio Hidráulico o Factor de } \\
\text { Forma } S=A / P\end{array}$ \\
\hline \multirow[t]{3}{*}{ W } & SB & 6 & 59 & 354 & 130 & 2.7 \\
\hline & HW & $3.20 *$ & 59 & 188.8 & 124.4 & 1.5 \\
\hline & $\mathrm{FW}$ & 3 & 59 & 177 & 124 & 1.4 \\
\hline \multirow[t]{3}{*}{$E$} & SB & $4.8^{*}$ & 19 & 91.2 & 47.6 & 1.9 \\
\hline & HW & $5.1^{*}$ & 19 & 96.9 & 48.2 & 2 \\
\hline & FW & 5 & 19 & 95 & 48 & 2 \\
\hline
\end{tabular}

Tabla 7. Determinación del radio hidráulico de cada una de las caras analizadas. (* se considera para las superficie inclinadas su verdadera dimensión).

Table 7. Determination of the hydraulic radius of each stope assessed (* it is considered the inclined stope as the true dimension). 


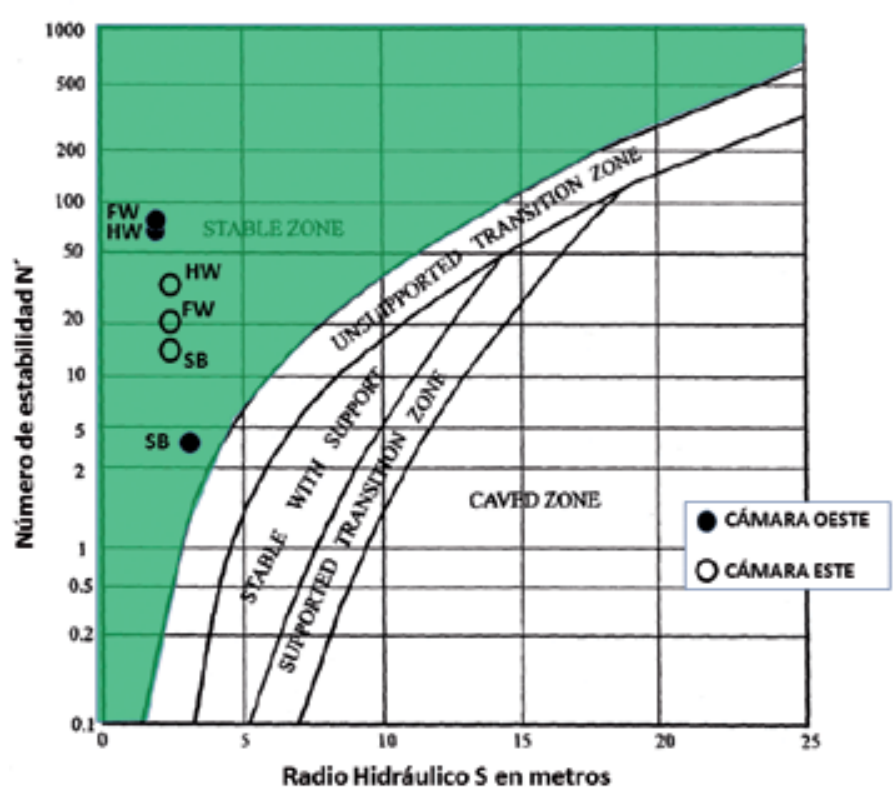

Figura 10. Gráfico de estabilidad que muestra las zonas de terreno estable, hundido y el que requiere sostenimiento. Tomado de Potvin (1988) modificado por Nickson (1992).

Figure 10. Stability graph showing zones of stable ground, caving ground and ground requiring support. After Potvin (1988), modified by Nickson (1992).

\section{Resultados modelización numérica - análisis tensodeformacional}

Una vez realizada una aproximación a la estabilidad de las labores mediante métodos empíricos se efectuó un análisis tensodeformacional de la cámara de mayores dimensiones. Se empleó un programa de análisis mediante elementos de finitos en dos dimensiones (Phase 2 v8 de la casa rocscience) y la envolvente de rotura de Hoek-Brown generalizada. Sin embargo en algunas zonas concretas es posible que se produzcan movimientos importantes, especialmente de bloques y la plastificación de las zonas menos competentes en las cámaras más grandes.

Para caracterizar el material no se dispone más que de datos de levantamientos geomecánicos de campo: las mencionadas estaciones en el interior de la mina. El "Geological Strenght index" o GSI indice de resistencia geólogica se ha determinado de modo visual (Fig.11).

Los parámetros utilizados en la modelización numérica (criterio de Hoek y Brown y su linealización a parámetros de cohesion y friccion instantáneas de Mohr Coulomb se muestran en el gráfico incluido en la Fig. 12. Los parámetros esenciales del criterio de Hoek - Brown se han inferido de forma indirecta a partir de índices de campo: la resistencia a

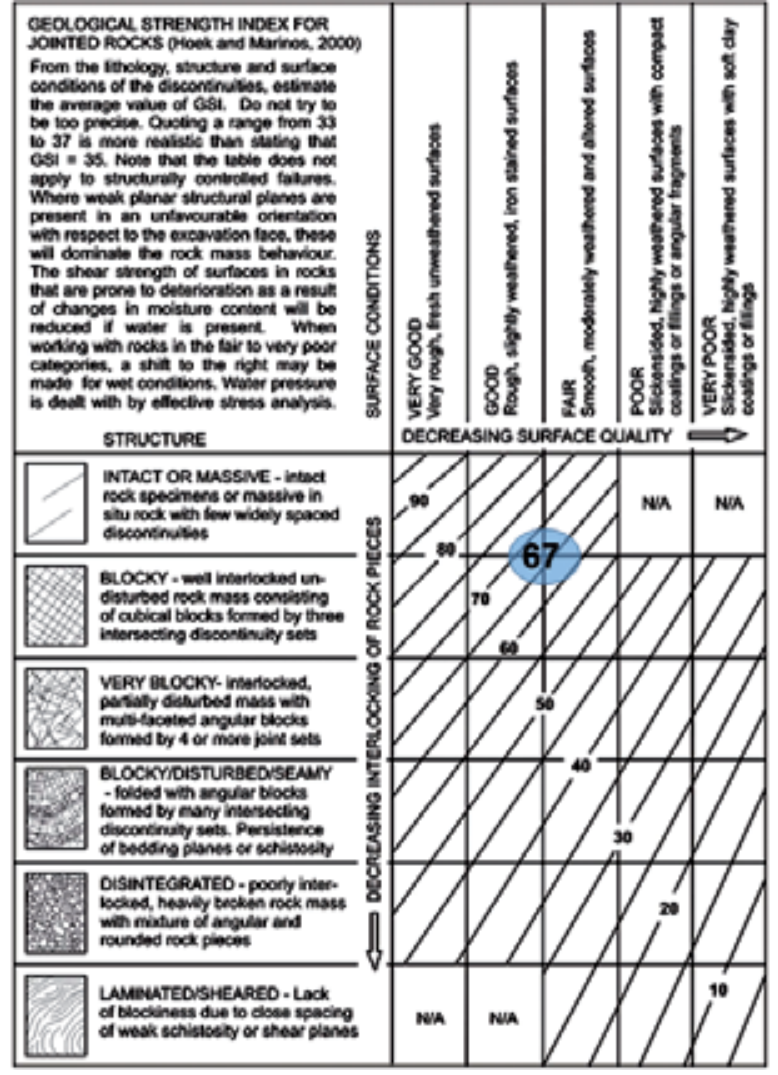

Figura 11. Determinacion gráfica - visual del GSI en la cámara de S'Argentera (basado en el gráfico de Marinos y Hoek, 2000, Carter y Marinos, 2014).

Figure 11. Graphic - visual determination of the GSI in S'Argentera stope (based on graph by Carter and Marinos,2014).
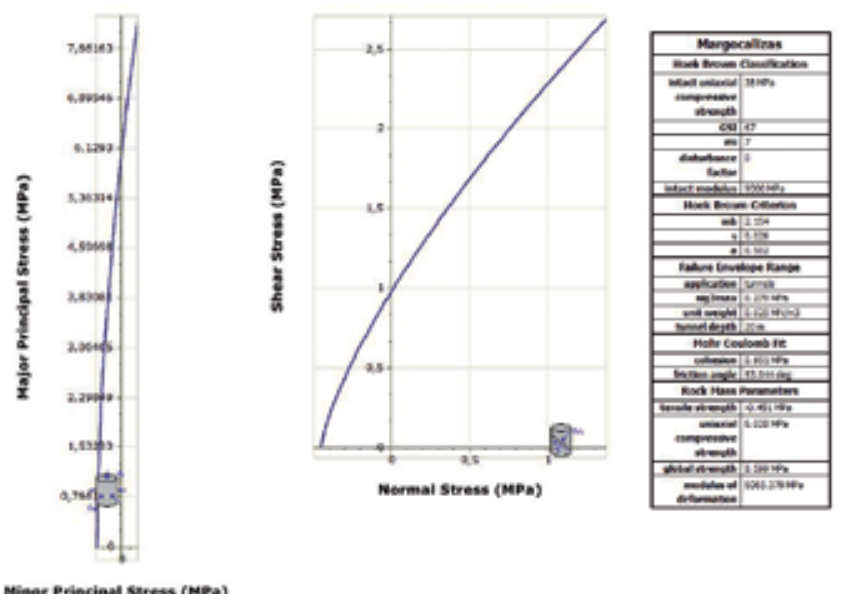

Miner Principat Stress (MPo)

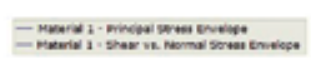

Figura 12. Envolvente de Hoek-Brown para el macizo rocoso -calizas margosas partiendo de datos de campo (utilizando programa RocataV5), donde se aprecian los parámetros de cálculo utilizados. Figure 12. Hoek-Brown material envelope for the rock mass-marl limestones using field data (using RocdataV5 Software) where parameters used can be checked. 
compresión simple uniaxial de la matriz rocosa $(\sigma c i)$ a partir de los rebotes del esclerómetro tipo N, el GSI visualmente (Fig. 11) y para el módulo de Young de la matriz, un valor propuesto por la propia hoja Rocdata en función del tipo de roca y la resistencia a compresión simple.

Se han modelizado los minados en dos dimensiones, con una tensión vertical gravitatoria tipo $\sigma v=\gamma \times \mathrm{h}$, con una $\mathrm{K}_{0}=1$ y condiciones de contorno con restricciones en el desplazamiento según el eje $Y$ en los límites verticales, libre en la superficie y restringidos $X e$ $Y$ en la base inferior. Los elementos considerados son de tipo triangular con tres nodos. Se han considerado cuatro fases incluidas la inicial y tres de excavación minera (Fig.13). Los resultados de elementos plastificados (Yielded Elements Fig. 14) muestran que existe cierta plastificación hacia el contacto hastial de techo - techo de la cámara desde la primera fase de excavación la cual progresa hacia la superficie y hacia cierta parte central del vano en la etapa final en la que toda la cámara está excavada: esta fue la situación al final de la explotación de la mina en 1909. En cuanto a los desplazamientos totales en superficie, éstos son en todo momento menores de $4 \mathrm{~cm}$, lo cual no supuso en tiempos de excavación mayor riesgo: pudo haber ligeros movimientos del terreno cuando la cámara se abrió del todo y ahora están del todo estabilizados. Los movimientos máximos en la cámara se dan en la parte central del vano y alcanzan $19 \mathrm{~cm}$ (Fig.15) en parte del techo que luego será excavado, lo cual refleja fielmente la realidad observada: estos bloques
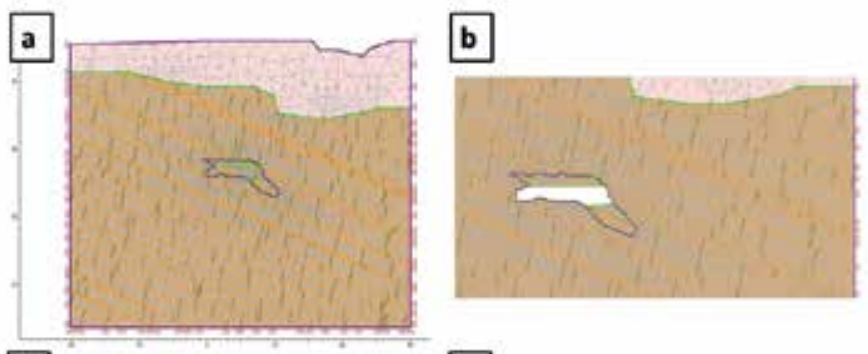

c

d
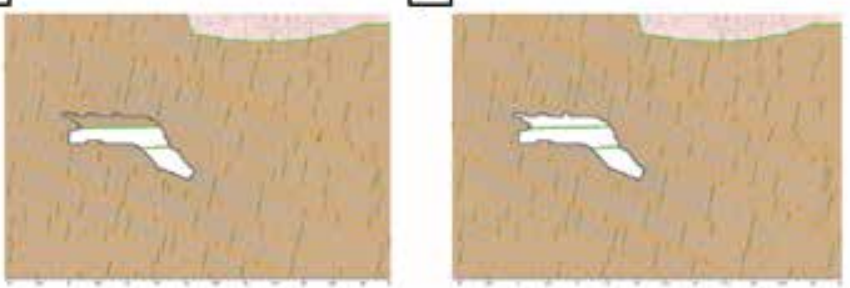

Figura 13. Modelo de elementos finitos mostrando las fases de cálculo consideradas - utilizando programa Phase2v8.

Figure 13. Finite element model showing the calculation stages considered - using Phase2v8 software. ya cayeron y actualmente no se aprecian grietas en el techo y sí bloques desprendidos de la época de explotación. Las tensiones máximas y concentración de tensiones: se producen tracciones en el centro del techo que propiciaron la caída de algún bloque posteriormente estabilizado por efecto arco de descarga.

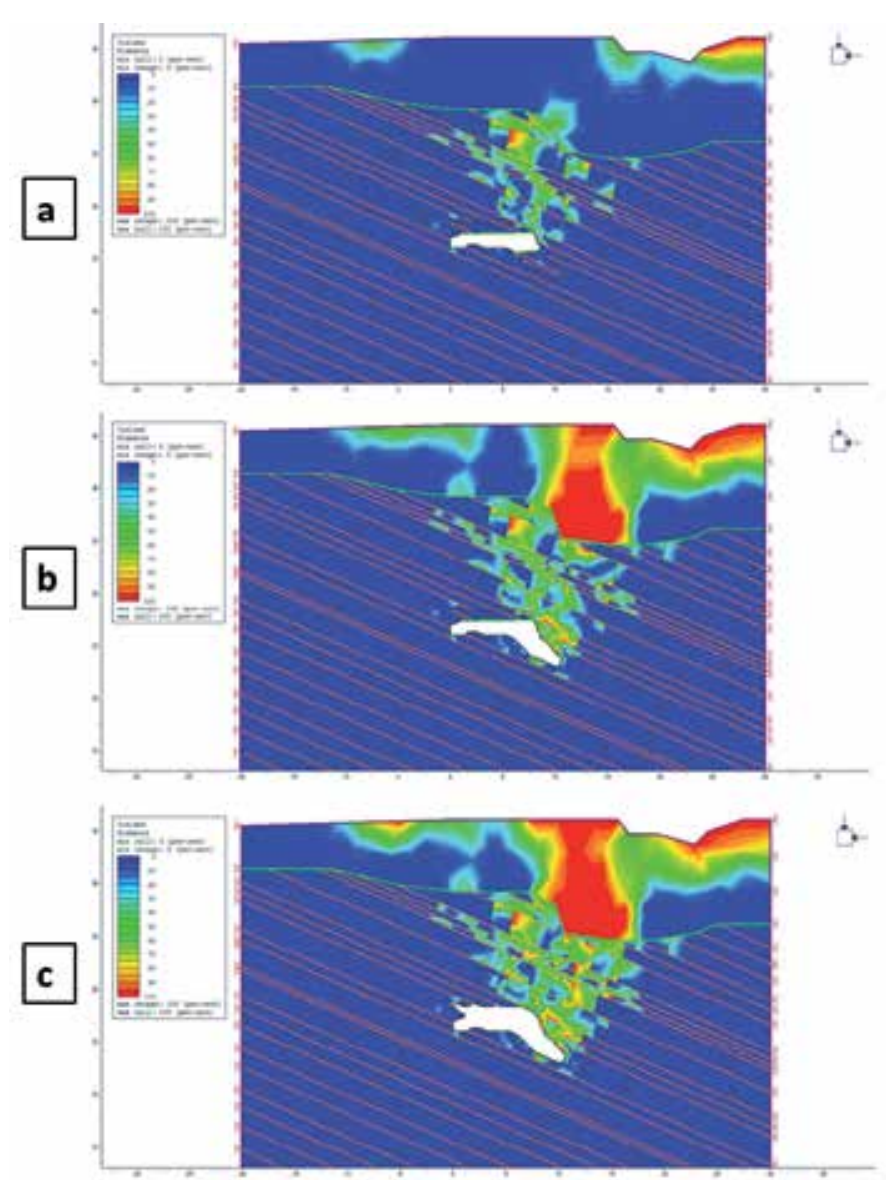

Figura 14. Elementos plastificados en las fases de cálculo - excavación de la cámara principal de las minas de SÁrgentera.

Figure 14. Yielded elements in the calculation stages - excavation of the main stope in the Argentera mines.

\section{Discusión}

En general las minas son estables, como atestigua el que sigan en pie tras más de cien años de abandono. Tanto los análisis empíricos -índice Barton, método gráfico y ancho escalado del pilar corona - como la modelización numérica, arrojan que la cámara es estable y no se espera que exista influencia sobre la superficie. Sin embargo algunos modelos y cálculos muestran que hay algunas inestabilidades locales que se aprecian en la mina y factores de seguridad 


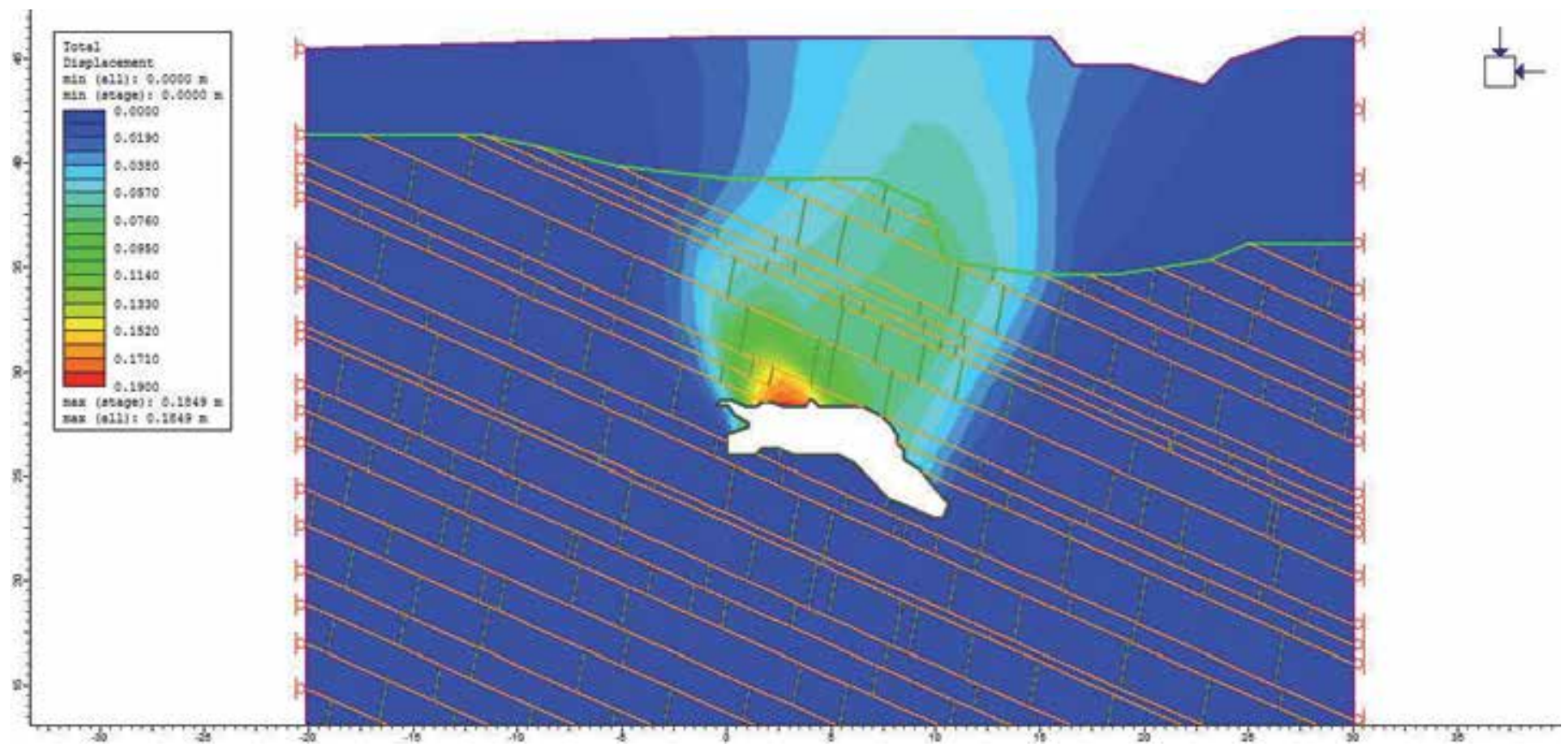

Figura 15. Desplazamientos totales con la cámara totalmente excavada (Phase2v8).

Figure 15. Total displacements in the stope totally excavated (Phase2v8).

algo justos, por lo que será conveniente un cierto monitoreo de posibles movimientos del terreno o subsidencias de la parcela y en especial de la carretera y, en caso de que se quiera hacer visitable el interior de las minas un estudio geotécnico en detalle.
Dado que se tienen buenos datos geométricos y geomecánicos (estaciones en el interior de las galerías) y geofísica para caracterizar el macizo- se ha podido elaborar un modelo geomecánico razonablemente fiel a la realidad. Este análisis aporta lógicamente

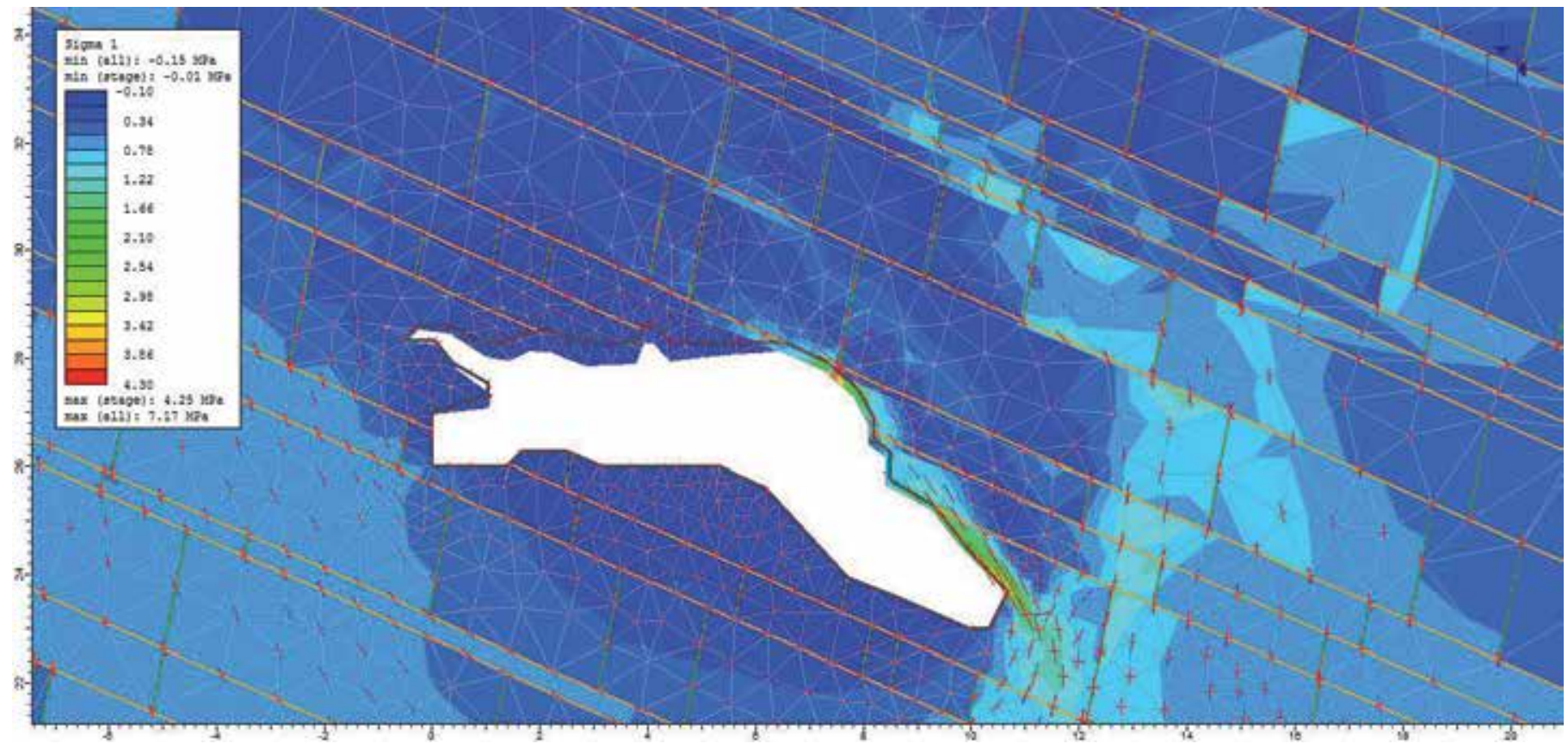

Figura 16. Tensión principal mayor y trayectoria de tensiones en la fase final de excavación (Phase2v8).

Figure 16. Major principal stress and stress trajectories in the final excavation stage (Phase2v8). 
Jordá-Bordehore, et al., 2017. Evaluación de la estabilidad de las labores y pilar... Boletín Geológico y Minero, 128 (1): 3-24

información más detallada sobre la propia cámara que las clasificaciones geomecánicas, si bien éstas permiten generalizar el problema y compararlo con un sinfín de casos a través de los gráficos empíricos, muchos de los cuales han ido retroalimentandos con casos prácticos (varios cientos) a lo largo de más de 30 años (Barton y Bieniawski 2008, Carter 2014).

Los métodos empíricos y la modelización numérica son técnicas complementarias y es recomendable aplicar ambas en los estudios geomecánicos. Los métodos empíricos están avalados por gran cantidad de casos como en el "ancho escalado" en el que es fácil que se hayan incluido en los gráficos algún caso semejante al que se esté analizando. Con una rápida verificación es fácil situar la mina objeto de estudio en la parte del gráfico "estable" o "hundido".

Los métodos de análisis empíricos suelen basarse en las clasificaciones geomecánicas. En el caso del ancho escalado y el método gráfico de estabilidad están basados ambos en el índice $\mathrm{Q}$. En la investigación realizada se ha visto como la aproximación mediante métodos empíricos y los diagramas de estabilidad son coherentes con las observaciones de campo en cada una de las cámaras: la cámara oeste de mejor calidad geomecánica permite la apertura de huecos mayores, mientras que la cámara este, de peor índi$\mathrm{ce}$, muestra algunas inestabilidades menores, y tanto en los diagramas de estabilidad del ancho escalado como en el método gráfico están representados por puntos más cercanos a la zona de inestabilidad.

Cada uno de los métodos analizados tiene en cuenta parámetros y aspectos diferentes por lo que es acertado el uso combinado de todos ellos. Lo más habitual en geomecánica es aplicar al menos dos métodos empíricos y posteriormente comprobar los resultados mediante análisis numérico (elementos finitos, diferencias finitas u otros menos habituales)

La prospección mediante la sísmica de refracción ha servido para dos temas: (a) se ha podido determinar la cobertera de la zona de suelo (y por tanto debe de considerarse como sobrecarga y no como pilar) y por tanto el espesor real del pilar corona; (b) los valores de índice $\mathrm{Q}$ obtenidos mediante mapeo y por geofísica son coherentes y por tanto las correlaciones existentes entre la $\mathrm{Vp}(\mathrm{m} / \mathrm{s})$ y el índice de calidad de roca Qc son de aplicación en este caso.

La evaluación de la estabilidad de la cámara mediante el índice $\mathrm{Q}$ arroja que, para el caso de que las cámaras fueran accesibles, se debería de aportar un cierto sostenimiento teniendo en cuenta el factor de seguridad requerido. En el presente trabajo se recomiendan unas subcategorías de Excavation Support Ratio, que tengan en cuenta el posible uso turístico del espacio subterráneo (cuevas y minas).
El índice $\mathrm{Q}$ y los gráficos que relacionan el ancho y la calidad de la roca es un tipo de análisis típico de túneles o galerías mineras donde se considera únicamente una sección y la longitud infinita. En el caso de cavernas y cámaras mineras este tipo de análisis resulta de una simplificación excesiva.

El método gráfico de estabilidad es el más apropiado para análisis de cámaras mineras puesto que tienen en consideración la geometría de la cámara en tres dimensiones (poliédrica). No es un método para análisis estrictamente bidimensional, sino que analiza un espacio poliédrico tridimensional a través de cada una de las caras que lo conforman. Tal y como se aprecia en la figura 10, todas las caras de las cámaras analizadas se ubican en el gráfico Número Estabilidad N' vs Radio Hidráulico en la zona estable, tal y como cabría esperar de unas labores mineras que llevan más de 100 años abiertas y solo presentan pequeñas inestabilidades puntuales de tipo cinemático (bloques).El techo de la cámara oeste, por ser la cara de mayores dimensiones es la que figura más cerca de la línea límite. Entre los hastiales, son los de la cámara este, que presentan peor calidad geotécnica los que se encuentran en la parte inferior de la zona estable. Es preciso señalar que el método gráfico, a diferencia del ábaco de $\mathrm{Q}$, no lleva implícito un factor de seguridad tipo ESR (Excavation Support Ratio). Es preciso ser muy cauto con este parámetro, que al fin y al cabo representa un factor de seguridad a añadir al vano que se analiza: en el caso de excavaciones mineras provisionales este factor "baja" la seguridad y considera para el análisis que el ancho es menor de lo que en realidad es y por tanto disminuye la seguridad; por el contrario, para obras civiles y cualquier excavación de carácter permanente, aumenta el ancho de análisis y por tanto penaliza y está del lado de la seguridad, con un factor corrector que puede ir desde 1,3 a 5 . Corresponde al proyectista elegir cuan conservador quiere ser y si sigue las pautas recomendadas en la tabla de ESR.

El análisis mediante ancho escalado para el pilar corona indica que éste se encuentra en un rango de seguridad apropiado, sin embargo, es recomendable, dado el uso turístico que se le pretende dar a la cavidad, que se realice o se instale un sistema básico de auscultación y levantamiento periódico de posibles inestabilidades. En todo caso, tanto para análisis de ancho escalado como para el método gráfico de estabilidad, el índice $\mathrm{Q}$ debe de calcularse antes, por lo que no está de más comparar los resultados con los que arrojan los gráficos convencionales del método de Barton.

El análisis tensodeformacional arroja resultados interesantes, tales como que pudo haber algunos 
asientos en superficie en la apertura máxima de las cámaras, que podrían ser responsables de algunas anomalías en la topografía del terreno, pero que actualmente y si no se amplían las cámaras no deberían de progresar: en 100 años éstas se han estabilizado. Los movimientos máximos del orden de varios centímetros que predicen los modelos están asociados a los movimientos de bloques tal y como puede apreciarse en la actualidad, la cámara Este aún posee bloques inestables que podrían movilizarse.

\section{Conclusiones}

Las evaluaciones por métodos empíricos y clasificaciones geomecánicas sugieren que las cámaras son estables y no se espera que exista influencia sobre la superficie.

- El análisis mediante el índice $Q$ de Barton señala que, según el ESR recomendado, es preciso un cierto sostenimiento en las cámaras. Este índice es empleado en los otros dos métodos empíricos.

- El análisis mediante el método gráfico sugiere que ambas cámaras son estables, si bien la Este y el techo de la Oeste que se encuentran más cerca de la zona de inestabilidad, requieren más atención.

- El análisis del pilar corona según el ancho escalado ubica al minado en la categoría más segura y por tanto de acceso libre. Sin embargo consideramos recomendable una mínima auscultación, máxime cuando pasa una carretera concurrida por encima.

- La nueva versión del programa CPillar (v 4.0) de Rocscience, recién desarrollada, incorpora un módulo de análisis empírico mediante el método del ancho escalado o "método de carter" que la versión anterior no incluía. Una línea de investigación futura podría consistir en, para el caso específico de análisis de pilares corona en minas abandonadas españolas, comparar los resultados aportados mediante el método empírico y los modelos elastoplasticos como el que implementa ese programa.

- El análisis tensodeformacional ha simulado las inestabilidades producidas en el centro de los vanos mayores y la posible presencia de pequeños asientos superficiales en tiempos de explotación de las minas. La simulación numérica no viene a refutar o aseverar los resultados de los factores de seguridad obtenidos mediante los métodos empíricos, dado que, en una simulación se obtiene no uno sino muchos, según la zona del hueco que se observe, mientras que en los métodos empíricos se obtiene un valor global, mediante gráficos intrínsecamente calibrados. El análisis numérico es complementario y aporta información sobre esfuerzos, desplazamientos y zonas plastificadas del terreno que no pueden obtenerse únicamente con las clasificaciones geomecánicas.

En el caso de los proyectos de puesta en valor del patrimonio minero para su aprovechamiento turístico es imprescindible un correcto análisis de las minas abandonadas, tanto de los propios huecos, como de su interacción con la superficie, de tal forma que se pueda concluir si se puede acceder a ellos o bien pasar por encima, si se debe restringir el acceso y/o reforzarlos o bien prohibir su acceso. La combinación de los métodos utilizados se ha mostrado claramente eficaz para la resolución del problema planteado y responder afirmativamente a las dos cuestiones planteadas ¿Es estable el minado? ¿Es posible el acceso público a la zona? Sin embargo, dados los factores de seguridad requeridos, es recomendable algunos medios de sostenimiento puntual en la cámara Oeste, mientras que la cámara Este es más inestable y las necesidades de refuerzo serían mucho mayores, recomendándose no acceder a ella, el pilar corona requeriría un monitoreo simple. Las clasificaciones geomecánicas y los métodos empíricos son herramientas muy potentes para el análisis de minados abandonados, pues con unas pocas medidas tanto de dimensiones como de parámetros geomecánicos (pues a menudo el acceso a estos minados está restringido) es factible hacer una evaluación certera de la estabilidad, o cuanto menos saber en qué zona del gráfico se sitúa: estable o inestable (hundimiento). En muchos casos los métodos empíricos son el mejor compromiso entre economía, tiempo de análisis y disponibilidad de datos veraces y están avalados por un histórico de casos, donde es posible que se haya incluido algún caso semejante.

\section{Agradecimientos}

Agradecemos de manera especial al Proyecto Prometeo de la Secretaría de Educación Superior, Ciencia, Tecnología e Innovación de la República del Ecuador por su patrocinio en este trabajo.

La toma de datos de campo de esta investigación ha sido llevada a cabo entre los años 2011 y 2014 gracias a las facilidades de Juan Escandell de Ses Mines de S'Argentera. Los análisis geotécnicos de gabinete, software e interpretación de resultados han sido realizados en la Facultad de Ingeniería en Ciencias de la Tierra, de la Escuela Superior Politécnica del Litoral (ESPOL) de Guayaquil, Ecuador. 


\section{Referencias}

Arribas, A. y Moro, C. 1985. Mineralizaciones españolas de $\mathrm{Pb}-\mathrm{Zn}$ asociadas a fenómenos cársticos en rocas triásicas y jurásicas. Stvdia Geologica Salmanticensia, 21, 125-151.

Barton, N. 2002. Some new Q-value correlations to assist in site characterisation and tunnel design. International Journal of Rock Mechanics and Mining Sciences, 39, 2002, 185-216.

Barton, N., Lien, R. and Lunde, J. 1974. Engineering classification of rock masses for the design of tunnel support. Rock Mechanics. 6: 4: 189-236.

Barton, N. and Bieniwaski, Z.T. 2008. RMR and Q: Setting records. Tunnels and Tunnelling International, February 2008, 26-29.

Barton, N.R. and Choubey, V. 1977. The shear strength of rock joints in theory and practice. Rock Mech. 10 (1-2) 1-54.

Barton, N.R. and Bandis, S.C. 1982. Effects of block size on the shear behaviour of jointed rock. $23^{\text {rd }}$ US Symp on rock mechanics, Berkeley, 739-760.

Barton, N.R. and Bandis, S.C. 1990. Review of predictive capabilities of JRC-JCS model in engineering practice. In rock joints, proc. int. symp. on rock joints. Loen. Norway (eds. N. Barton and O. Stephansson). 603-610. Rotterdam: Balkema.

Barton, N. and Bieniawski, Z.T. 2008. RMR and Q - Setting records straight. Tunneling abd tunnelling international, Feb 2008, pp. 26-29.

Bieniawski, Z. T.1973. Engineering Classification of jointed rock masses. Trans S Afr Int Civ Engrs 15, 335-344.

Bieniawski, Z.T. 1989. Engineering Rock Mass Classification. Wiley, Chichester, $251 \mathrm{pp}$.

Bieniawski, Z.T. 2011. Errores en la aplicación de las clasificaciones geomecánicas y su corrección. Conferencia magistral Adif - Geocontrol, $35 \mathrm{pp}$.

Carter, T.G. and Miller R.I. 1995. Crown pillar risk assessment - cost effective measures for mine closure remediation planning. Trans.Inst.Min.Metl. 104, 41-57.

Carter, T.G., Cottrell, B.E., Carvalho, J.L. y Steed, C.M. 2008. Logistic regression Improvements to the scale span method for dimensioning surface crown pillars over civil or mining openings. American Rock Mechanics Association, Proceedings, 2008.

Carter, T.G. 2014. Shourt course - Crown pillar stability assessment. Course notes and guidelines. 1er Congreso Internacional de Diseño de Mina por Métodos Empíricos, Lima, Perú, junio 2014, 34 pp.

Cornejo, L. y Salvador, E. 1996. Manual de túneles interurbanos de carretera. Gobierno Vasco, 211 pp.

Coulson J.H. 1972. Shear Strengh of flat surfaces in rock stability of rock slopes. In: Cording EE.J. (ed) Procedings of $13^{\text {th }}$ Symposium on Rock Mechanics. American Society of civil engineers, New York, 77-105.

Escandell Serra, J. 2001. Ses Mines de I'Argentera. Projecte per al seu aprofitament turístic, en: J. Carles Cirer Costa (ed.), Estudis sobre el turisme a Eivissa i Formentera 2. Ibiza, 41-48.
Golder Associates. 1990. Report 881-1739 to CanMet on "Crown Pillar Stability Back Analysis". Report \#234408-9074/01-SQ, Canmet, 90 pp.

Helm, P.R, Davie, C.T. and Glendinng, S. 2013. Numerical modelling of shallow abandoned mine working subsidence affecting transport infrastructure. Engineering Geology. Vol 154, 6-19

Hoek, E. 2007. Practical Rock Engineering. Set of course notes. Shear strength of discontinuities. www.rocscience.com/education/hoeks_corner (4-dec-2014)

Hoek, E. and Diederichs, M.S. 2006. Empirical estimation of rock mass modulus. International Journal of Rock Mechanics and Mining Sciences 43, 203-215.

Hoek, E., Carranza Torres, C. and Corkum, B. 2002. HoekBrown failure criterion-2002 edition. Proceedings of NARMS-Tac, 267-273.

Hoek, E., Kaiser, P.K. and Bawden, W.F. 1995. Support of Underground Excavations in Hard Rock. Editor: Taylor and Francis, Fourth Print 2005, 215 pp.

Jordá Bordehore, L. 2009. La minería de los metales en la provincia de Madrid: patrimonio minero y puesta en valor del espacio subterráneo. Tesis doctoral, Universidad Politécnica de Madrid, inédita. 780 pp + anejos (180 pp. de fichas y 11 planos).

Jordá Bordehore, L., Jordá Bordehore, R. y Fernández Amo, F.J. 2010. Contribución al conocimiento geotécnico de la Mina Monchi de cara a su musealización. Ingeopres $\mathrm{n}^{\circ} 195,28-33$

Jordá Bordehore, L. y Jordá Bordehore, R. 2009. Estudio geotécnico preliminar de la mina de plata abandonada de Bustarviejo (Sierra de Guadarrama, Madrid). En: J.J. Durán y J, López Martinez (Eds.), Cuevas turísticas, cuevas vivas pp. 285-293. Madrid. Asociación de Cuevas Turísticas Españolas. 414 pp. Actas /l Congreso Español de Cuevas Turísticas, Santander, Cantabria 1618 de octubre de 2008.

Jordá Bordehore, L., Heinrich Hermanns, M. y Jordá Bordehore, R. 2011. Apuntes para el conocimiento de las minas de plomo argentífero de S'Argentera (Ibiza) en los siglos XIX y XX. Re Metallica, 17, 1-12.

Marinos, P. and Hoek, E. 2000. GSI - A geologically friendly tool for rock mass strength estimation. Proc. GeoEng2000 Conference, Melbourne. 1422-1442.

Martin, C.D., Kaiser, P.K. and McCreath, D.R., 1999. HoekBrown parameters for predicting the depth of brittle failure around tunnels. Can. Geotech J., 36(1), 136-151.

N.G.I. 2013. Using the $Q$ system. Handbook. Rock mass classification and support design. Ed. NGI, Oslo, Norway. 57 pp.

Nickson, S.D. 1992. Cable support guidelines for underground hard rock mine operations, MSc, thesis, Dept. Mining and Mineral Processing, University of British Columbia.

Priest, S.D. and Hudson, J.A. 1976. Discontinuity spacings in Rock. International Journal of Rock Mechanics and Mining Sciences, 13: 135-148

Potvin, Y. 1988. Empirical open stope design in Canada. PhD., thesis, Dept.Mining and Mineral Processing, University of British Columbia. 
I.T.G.E. 1991. Mecánica de Rocas aplicada a la minería metálica subterránea, Ed. Instituto Tecnológico Geominero, Madrid, $334 \mathrm{pp}$.

I.T.G.E. 1995. Caracterización de estructuras subterráneas artificiales. Aplicación a un caso concreto (estructuras subterráneas, Asturias II) Aplicación mina Villabona. Informe Goetécnico y de subsidencia. Resultados y análisis de los ensayos de laboratorio. Convenio específico ITGE Principados de Asturias, 38 pp. Informe Interno accesible online: http://info.igme. es/SidPDF\%5C039000\%5C311\%5CInforme\%20geologico\%5C39311_0007.pdf (25/05/2015)

Rocscience Inc. 2011, Phase2 Version 8.0 - Finite Element Analysis for Excavations and Slopes. www.rocscience. com, Toronto, Ontario, Canada.
Rocscience Inc. 2005, RocData Version 4.0 - Rock, Soil and Discontinuity Strength Analysis. www.rocscience.com, Toronto, Ontario, Canada.

Rocscience Inc. 2001, Dips version 5.0 Graphical and statistical analysis of orientation data. www.rocscience.com Toronto, Ontario, Canada.

Yong-Bok Jung, Won-Kyong Song, Dae-Sung Cheon, Dong-Kil Lee and Joo-Young Park. 2014. Simple method for the identification of subsidence susceptibility above underground coal mine in Korea. Engineering Geology. Vol 178, pp. 121-131.

Waltham T., Parh H.D., Suh, J., Yu, M.H., Kwon, H.H. and Bang, K.M. 2011. Collapses of old mines in Korea. Engineering Geology. Vol 118, issue 1-2., pp.29-36.

Recibido: julio 2015

Revisado: septiembre 2015

Aceptado: septiembre 2015

Publicado: marzo 2017 
